\title{
THE TRANSFER OF RADIATION IN STELLAR ATMOSPHERES
}

\section{S. CHANDRASEKHAR}

1. Introductory remarks. The advances in the various branches of theoretical physics have often resulted in the creation of new mathematical disciplines: disciplines which, in their way, are as characteristic of the subjects as the physical phenomena they are devoted to. That several of the earlier Gibbs lectures should illustrate this point is not surprising since the example provided by the scientific writings of Gibbs is indeed the most striking in this connection. With your permission, I would like to illustrate the same thing this evening, on a more modest level, by considering the recent advances in theoretical astrophysics. More specifically, I should like to show how astrophysical studies relating to the transfer of radiation in stellar atmospheres have led to some characteristic developments in the theory of integrodifferential and functional equations.

2. The physical problem. In a general way, the preoccupation of the astrophysicist with the transport of radiant energy in an atmosphere which absorbs, emits and scatters radiation is not difficult to understand. For, it is in the characteristics of the radiation emergent from a star-in its variation over the stellar disc, and in its distribution with wavelength through the spectrum-that he has to seek for information concerning the constitution and structure of the stellar atmosphere.

3. Definitions. Intensity. Now, the basic equation which governs the transfer of radiation in any medium is the so-called equation of transfer. This is the equation which governs the variation of radiant intensity in terms of the local properties of the medium. But, first, I should explain that for most purposes it is sufficient to characterize the radiation field by the specific intensity $I$; in terms of this quantity the amount of radiant energy crossing an element of area $d \sigma$, in a direction inclined at an angle $\vartheta$ to the normal, and confined to an element of solid angle $d \omega$, and in time $d t$ can be expressed in the form

$$
I \cos \vartheta d \sigma d \omega d t \text {. }
$$

Accordingly, to characterize a radiation field completely, the specific

The twentieth Josiah Willard Gibbs lecture delivered at Swarthmore, Pennsylvania, December 26, 1946, under the auspices of the American Mathematical Society; received by the editors January 13, 1947. 
intensity will have to be defined at every point and for every direction through a point.

4. The equation of transfer. To establish the equation which the intensity must satisfy in a stationary radiation field (that is, in a radiation field which is constant with time) we consider the equilibrium with respect to the transport of energy of a small cylindrical element of cross section $d \sigma$ and height $d s$ in the medium. From the definition of intensity, it follows that the difference in the radiant energy crossing the two faces normally, in a time $d t$ and confined to an element of solid angle $d \omega$, is

$$
\frac{d I}{d s} d s d \sigma d \omega d t
$$

Here $I$ denotes the specific intensity in the direction of $s$. This difference (2) must arise from the excess of emission over absorption of radiant energy in time $d t$ and the element of solid angle $d \omega$ considered. Now the absorption of radiation by an element of mass is expressed in terms of the mass absorption coefficient denoted by $\kappa$, such that, of the energy $I d \sigma d \omega d t$ normally incident on the face $d \sigma$, the amount absorbed in time $d t$ is

$$
\kappa \rho d s \times I d \sigma d \omega d t,
$$

where $\rho$ denotes the density of the material. Similarly, the emission coefficient $j$ is defined in such a way that the element of mass $\rho d \sigma d s$ emits in directions confined to $d \omega$ and in time $d t$ an amount of radiant energy given by

$$
j \rho d \sigma d s d \omega d t \text {. }
$$

It should be noted that, according to our definition, the emission coefficient $j$ can very well depend on the direction at each point, differing in this respect from the absorption coefficient which is a function of position only.

From our earlier remarks and the expressions (3) and (4) for absorption and emission, we have the equation of transfer

$$
\frac{d I}{\rho d s}=-\kappa I+j
$$

expressing the conservation of radiant energy.

5. The equation of transfer for plane-parallel atmospheres. The 
source function. In our further discussion we shall restrict ourselves to transfer problems in a semi-infinite plane-parallel atmosphere (Fig. 1). Let $z$ measure distances normal to the plane of stratification and $\vartheta$ and $\varphi$ the corresponding polar angles. The specific intensity $I$ in such an atmosphere will, in general, depend on all three variables, $z, \vartheta$

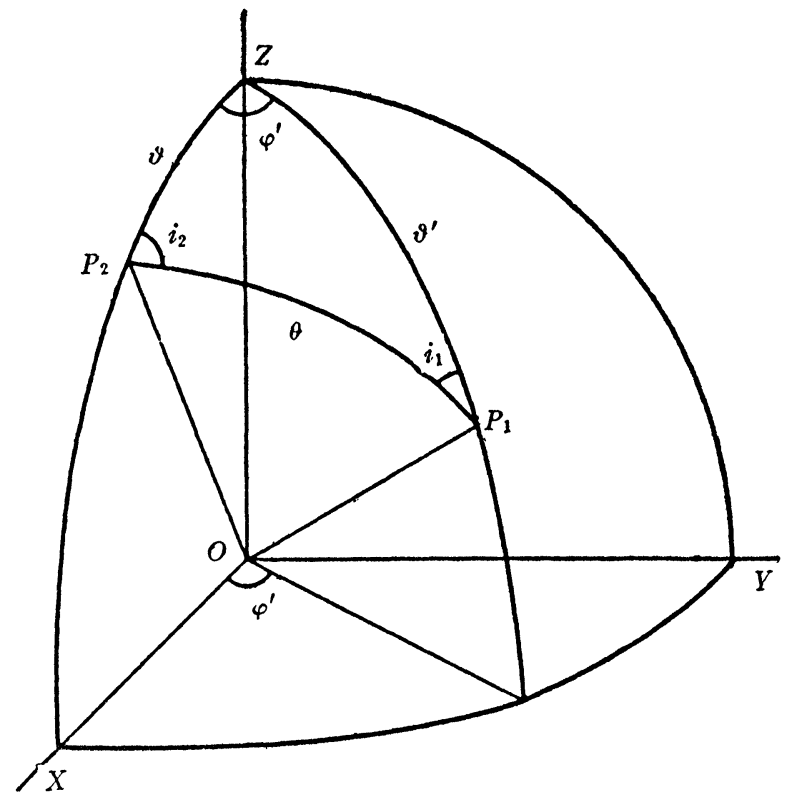

FIG. 1.

and $\varphi$. For such an atmosphere the equation of transfer can be written in the form

$$
\cos \vartheta \frac{d I}{\rho d z}=-\kappa I+j .
$$

It is convenient at this stage to introduce the optical thickness

$$
\tau=\int_{z}^{\infty} \kappa \rho d z
$$

measured from the boundary inward as our variable instead of $z$. In terms of $\tau$, the equation of transfer becomes

$$
\cos \vartheta \frac{d I}{d \tau}=I(\tau, \vartheta, \varphi)-\Im(\tau, \vartheta, \varphi),
$$


where we have written

$$
\Im=\frac{j}{\kappa} .
$$

This is the source function.

6. The source function for a scattering atmosphere. The phase function. Different physical problems lead to different functional dependences of the source function on $I$. To illustrate the nature of such functional dependences we shall consider the case of a scattering atmosphere. In such an atmosphere, absorption arises simply on account of the fact that when a pencil of radiation is incident on an element of gas, a determinate part of the radiant energy is scattered in other directions. In the same way, the radiation in any given direction can be reinforced by the scattering of radiation from other directions into the particular direction considered. It is now evident that, to formulate quantitatively the concept of scattering, we must specify the angular distribution of the scattered radiation when a pencil of radiation is incident on an element of gas. This angular distribution is generally given by a phase function $p(\cos \Theta)$ in such a way that

$$
p(\cos \Theta) \frac{d \omega}{4 \pi}
$$

governs the probability that radiation will be scattered in a direction inclined at an angle $\Theta$ with the direction of incidence. If, as we have assumed, scattering is the only process by which radiation and matter interact with each other, then it is evident that

$$
\int p(\cos \Theta) \frac{d \omega}{4 \pi}=1
$$

when the integration is extended over the complete sphere. The corresponding source function is

$$
\begin{aligned}
\Im(\tau, \vartheta, \varphi)=\frac{1}{4 \pi} \int_{0}^{\pi} \int_{0}^{2 \pi} & I\left(\tau, \vartheta^{\prime}, \varphi^{\prime}\right) p\left(\cos \vartheta \cos \vartheta^{\prime}\right. \\
& \left.+\sin \vartheta \sin \vartheta^{\prime} \cos \left[\varphi^{\prime}-\varphi\right]\right) \sin \vartheta^{\prime} d \vartheta^{\prime} d \varphi^{\prime} .
\end{aligned}
$$

When the scattering is isotropic

$$
p(\cos \Theta) \equiv 1
$$

and the source function reduces to 


$$
\Im(\tau)=\frac{1}{4 \pi} \int_{0}^{\pi} \int_{0}^{2 \pi} I\left(\tau, \vartheta^{\prime}, \varphi^{\prime}\right) \sin \vartheta^{\prime} d \vartheta^{\prime} d \varphi^{\prime} .
$$

Next to this isotropic case, greatest interest is attached to Rayleigh's phase function

$$
p(\cos \Theta)=\frac{3}{4}\left(1+\cos ^{2} \Theta\right) .
$$

In general, we may, however, suppose that the phase function can be expanded as a series in Legendre polynomials of the form

$$
p(\cos \Theta)=\sum \varpi_{l} P_{l}(\cos \Theta)
$$

where $\varpi_{0}=1$ and $\varpi_{l}(l \neq 0)$ are some constants. (The condition $\varpi_{0}=1$ follows from equation (11).)

7. Phase-functions with absorption. Our foregoing remarks strictly apply to the case of perfect scattering; that is, to cases in which the interaction between matter and radiation results only in the transformation of radiation in one direction into radiation in other directions. However, cases are known in which, in the process of scattering, a certain amount of the radiation is consumptively transformed into other forms of energy. In such cases, we can still describe the scattered radiation by a phase function $p(\cos \Theta)$; but the "normalizing condition" (11) will no longer be satisfied and we shall have instead

$$
\int p(\cos \Theta) \frac{d \omega}{4 \pi}=\lambda
$$

The quantity $\lambda$ defined in this way is called the albedo. Formally, this does not introduce anything very essential, as the source function will continue to be given by equation (12).

The case

$$
p(\cos \Theta)=\lambda(1+x \cos \Theta) \quad(-1<x<1)
$$

is of particular interest for the analysis of planetary illumination.

8. The problem of a semi-infinite plane-parallel atmosphere with a constant net flux. Returning to the case of a perfectly scattering atmosphere, we observe that the equation of transfer

$$
\cos \vartheta \frac{d I(\tau, \vartheta, \varphi)}{d \tau}=I(\tau, \vartheta, \varphi)-\frac{1}{4 \pi} \int_{0}^{\pi} \int_{0}^{2 \pi} I\left(\tau, \vartheta^{\prime}, \varphi^{\prime}\right) p(\cos \Theta) d \omega^{\prime},
$$

where

$$
\cos \Theta=\cos \vartheta \cos \vartheta^{\prime}+\sin \vartheta \sin \vartheta^{\prime} \cos \left(\varphi^{\prime}-\varphi\right)
$$


admits the first integral

$$
\frac{d}{d \tau} \int_{0}^{\pi} \int_{0}^{2 \pi} I(\tau, \vartheta, \varphi) \cos \vartheta \sin \vartheta d \vartheta d \varphi=0
$$

or

$$
\pi F=\int_{0}^{\pi} \int_{0}^{2 \pi} I(\tau, \vartheta, \varphi) \cos \vartheta \sin \vartheta d \vartheta d \varphi=\text { constant }
$$

The integral representing $F$ is proportional to the net flux of radiant energy crossing unit area in the plane of stratification; and this is a constant for a perfectly scattering atmosphere. Because of this constancy of the net flux, a type of transfer problem which arises in connection with such perfectly scattering atmsopheres is that of a plane-parallel semi-infinite atmosphere with no incident radiation, and a constant net flux $\pi F$ through the atmosphere normal to the plane of stratification. It is evident that for these problems, solutions of the appropriate equations of transfer must be sought which depend only on $\tau$ and $\vartheta$, that is, solutions which exhibit rotational symmetry about the z-axis. Further, in these problems, the greatest interest is attached to the angular distribution of the emergent radiation $I(0, \mu)$ at $\tau=0$ and for $\mu>0$.

Finally, we may note that for the cases of isotropic scattering and scattering in accordance with Rayleigh's phase function, the equations of transfer have explicitly the forms

$\mathbf{I}$

$$
\mu \frac{d I(\tau, \mu)}{d \tau}=I(\tau, \mu)-\frac{1}{2} \int_{-1}^{+1} I\left(\tau, \mu^{\prime}\right) d \mu^{\prime},
$$

and

II

$$
\begin{aligned}
\mu \frac{d I(\tau, \mu)}{d \tau}=I(\tau, \mu)-\frac{3}{16}[ & \left(3-\mu^{2}\right) \int_{-1}^{+1} I\left(\tau, \mu^{\prime}\right) d \mu^{\prime} \\
& \left.+\left(3 \mu^{2}-1\right) \int_{-1}^{+1} I\left(\tau, \mu^{\prime}\right) \mu^{\prime 2} d \mu^{\prime}\right]
\end{aligned}
$$

In the foregoing equations we have written $\mu$ for $\cos \vartheta$.

We require solutions of the integro-differential equations (I) and (II) which satisfy the boundary condition

$$
I(0, \mu)=0 \quad \text { for }-1<\mu<0 .
$$


9. The problem of diffuse reflection. Another type of transfer problem which arises quite generally (that is, also in cases when $\lambda$ is different from unity) is that relating to the phenomenon of diffuse reflection. In these contexts we are principally interested in the angular distribution $I\left(\vartheta, \varphi ; \vartheta_{0}, \varphi_{0}\right)$ of the radiation diffusely reflected when a parallel beam of radiation of net flux $\pi F$ per unit area normal to itself is incident on the atmosphere in some specified direction $\left(\pi-\vartheta_{0}, \varphi_{0}\right)$.

One general remark relating to problems of diffuse reflection may be made here. It is that, at any level, we may distinguish between the reduced incident radiation $\pi F e^{-\tau \sec \vartheta_{0}}$ which penetrates to the depth $\tau$ without suffering any scattering or absorbing mechanism, and the diff use radiation field which arises in consequence of multiple scattering. We shall characterize this diffuse radiation field by $I(\tau, \mu, \varphi)$. Making this distinction between these two fields of radiation, we may write the equation of transfer for problems of diffuse reflection in the form

$$
\begin{aligned}
\cos \vartheta & \frac{d I(\tau, \vartheta, \varphi)}{d \tau}=I(\tau, \vartheta, \varphi)-\frac{1}{4 \pi} \int_{0}^{\pi} \int_{0}^{2 \pi} I\left(\tau, \vartheta^{\prime}, \varphi^{\prime}\right) \\
\times & p\left(\cos \vartheta \cos \vartheta^{\prime}+\sin \vartheta \sin \vartheta^{\prime} \cos \left[\varphi^{\prime}-\varphi\right]\right) \sin \vartheta^{\prime} d \vartheta^{\prime} d \varphi^{\prime} \\
& -\frac{1}{4} F e^{-\tau \sec \vartheta_{0}} p\left(-\cos \vartheta \cos \vartheta_{0}+\sin \vartheta \sin \vartheta_{0} \cos \left[\varphi_{0}-\varphi\right]\right)
\end{aligned}
$$

For the cases of isotropic scattering, and scattering in accordance with the phase functions (15) and (18), we have the equations

III

$$
\mu \frac{d I(\tau, \mu)}{d \tau}=I(\tau, \mu)-\frac{1}{2} \int_{-1}^{+1} I\left(\tau, \mu^{\prime}\right) d \mu^{\prime}-\frac{1}{4} F e^{-\tau / \mu_{0}}
$$

$$
\begin{aligned}
& \mu \frac{d I(\tau, \vartheta, \varphi)}{d \tau}=I(\tau, \vartheta, \varphi) \\
& -\frac{\lambda}{4 \pi} \int_{0}^{\pi} \int_{0}^{2 \pi} I\left(\tau, \vartheta^{\prime}, \varphi^{\prime}\right)\left[1+x\left(\cos \vartheta \cos \vartheta^{\prime}\right.\right. \\
& \left.\left.+\sin \vartheta \sin \vartheta^{\prime} \cos \left[\varphi^{\prime}-\varphi\right]\right)\right] \sin \vartheta^{\prime} d \vartheta^{\prime} d \varphi^{\prime} \\
& -\frac{1}{4} \lambda F e^{-\tau \sec \vartheta_{0}}\left[1+x\left(-\cos \vartheta \cos \vartheta_{0}+\sin \vartheta \sin \vartheta_{0} \cos \varphi\right)\right]
\end{aligned}
$$

and 


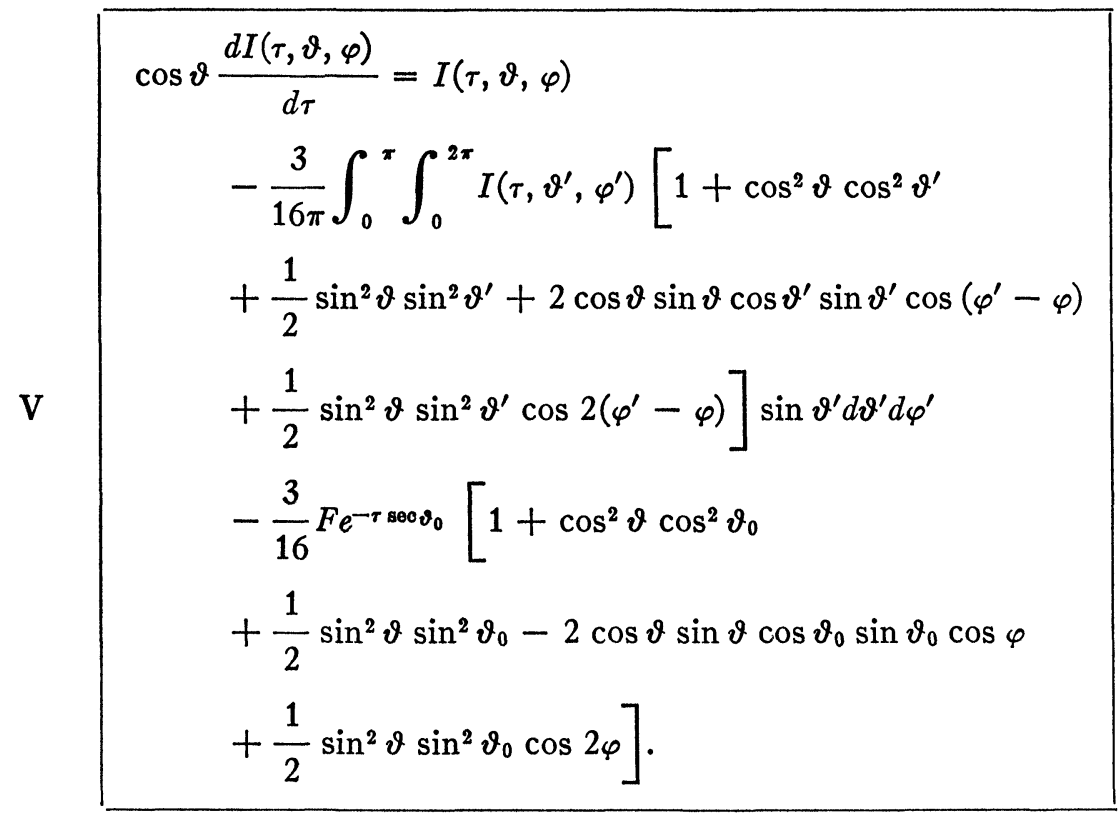

And again we require solutions of these equations which satisfy the boundary condition (23).

It appears from the foregoing equations that for problems of diffuse reflection which arise in practice, we can expand $I(\tau, \mu, \varphi)$ in a finite Fourier series of the form

$$
I(\tau, \mu, \varphi)=\sum_{m=0}^{N} I^{(m)}(\tau, \mu) \cos m \varphi .
$$

And finally, it should be remarked that in the problem of diffuse reflection by a semi-infinite atmosphere, greatest interest is attached to the scattering function $S\left(\mu, \varphi ; \mu_{0}, \varphi_{0}\right)$ which is related to the reflected intensity $I(0, \mu, \varphi)$ according to the equation

$$
I(0, \mu, \varphi)=\frac{1}{4 \mu} F S\left(\mu, \varphi ; \mu_{0}, \varphi_{0}\right) .
$$

Defined in this manner $S\left(\mu, \varphi ; \mu_{0}, \varphi_{0}\right)$ must be symmetrical in the pair of variables $(\mu, \varphi)$ and $\left(\mu_{0}, \varphi_{0}\right)$. This is required by a principle of reciprocity due to Helmholtz.

10. The equations of transfer incorporating the polarization of the scattered radiation in accordance with Rayleigh's law. The axially symmetric case. The transfer problems which we have described in 
the preceding sections do not exhaust all the physical possibilities that may arise. For example, an important factor which we have not included in our discussion so far concerns the state of polarization of the radiation field. For many problems, this must be taken into account before we may be said to have an adequate physical description of the phenomenon under consideration, since, on scattering, light in general gets polarized. On Rayleigh's classical laws for instance, when an initially unpolarized beam is scattered in a direction inclined at an angle $\Theta$ with the direction of incidence, it becomes partially plane polarized with a ratio of intensities $1: \cos ^{2} \Theta$ in directions perpendicular, respectively, parallel to the plane of scattering. (This is the plane which contains the directions of the incident and the scattered light.) It is, therefore, apparent that the diffuse radiation field in a scattering atmosphere must be partially polarized. The question immediately arises as to how best we can characterize the radiation field under these conditions in order that the relevant equations of transfer may be most conveniently formulated. This is a fundamental question: on its answer will depend the solution of a variety of problems, including the important one of the illumination and the polarization of the sunlit sky - a problem which was in fact first considered in a general way by Rayleigh in 1871 . It is, therefore, somewhat surprising to find that even the basic equations of the problem should not have been written down before the problem arose in an astrophysical context earlier this year. Perhaps I may briefly explain the particular astrophysical problem which gave the final impetus for formulating and solving transfer problems in which the polarization characteristics of the radiation field are incorporated and allowance for the polarization of the scattered radiation is made:

It is now believed that in the atmospheres of stars with surface temperatures exceeding $15,000^{\circ} \mathrm{K}$, the transfer of radiation must be predominantly controlled by the scattering by free electrons. And, according to J. J. Thomson's laws for this process, scattering by free electrons must result in the partial polarization of the scattered radiation. Thomson's laws agree, in fact, with Rayleigh's as regards the angular distribution and the state of polarization of the scattered radiation.

Now, if our belief in the important role played by the Thomson scattering in the atmospheres of hot stars is correct, we should expect that the radiation emergent from the atmospheres of such stars should be partially polarized. The question of the degree of polarization to be expected under such conditions therefore becomes one of exceptional astrophysical interest. But, before we can answer this ques- 
tion, we must formulate the relevant equations of transfer, distinguishing the different states of polarization, and solve them! One circumstance, however, simplifies this particular problem. It is, that in a plane-parallel atmosphere with no incident radiation, the radiation field at any point must exhibit rotational symmetry about the $z$-axis; accordingly, the plane of polarization must be along the principal meridian (or, at right angles to it). In other words, under the conditions of our present problem, the specific intensities $I_{l}$ and $I_{r}$, referring respectively to the two states of polarization in which the electric vector vibrates in the meridian plane and at right angles to it, are sufficient to characterize the radiation field completely. And the relevant equations of transfer appropriate for this problem are found to be:

VI

$$
\begin{aligned}
\mu \frac{d I_{l}}{d \tau}=I_{l} & -\frac{3}{8}\left\{\int_{-1}^{+1} I_{l}\left(\tau, \mu^{\prime}\right)\left[2\left(1-\mu^{\prime 2}\right)+\mu^{2}\left(3 \mu^{\prime 2}-2\right)\right] d \mu^{\prime}\right. \\
& \left.+\mu^{2} \int_{-1}^{+1} I_{r}\left(\tau, \mu^{\prime}\right) d \mu^{\prime}\right\} \\
\mu \frac{d I_{r}}{d \tau}=I_{r} & -\frac{3}{8}\left\{\int_{-1}^{+1} I_{l}\left(\tau, \mu^{\prime}\right) \mu^{\prime 2} d \mu^{\prime}+\int_{-1}^{+1} I_{r}\left(\tau, \mu^{\prime}\right) d \mu^{\prime}\right\}
\end{aligned}
$$

In other words, we now have a pair of simultaneous integro-differential equations to solve with the boundary conditions

$$
I_{l}(0, \mu)=I_{r}(0, \mu)=0 \quad(-1<\mu<0) .
$$

11. The parametric representation of partially plane-polarized light. The vector form of the equation of transfer. Returning to the general problem of a partially polarized radiation field, we at once realize that the intensities $I_{l}$ and $I_{r}$ are not sufficient to characterize the radiation field completely. A third quantity is needed which will determine the plane of polarization. But the inclination of the plane of polarization itself to the meridian plane (for example) would be a most unsuitable parameter to choose: for, a proper parametric representation of partially polarized light should not only provide a complete set of variables to describe the radiation field, but also enable a convenient formulation of the rule of composition of a mixture of independent polarized streams in a given direction into a single partially polarized stream. On consideration it appears that the most convenient third variable to choose is a quantity $U$ also of the dimensions of intensity and in terms of which the inclination, $\chi$, of the plane of polarization to the direction to which $I_{l}$ refers is giver by 


$$
\chi=\frac{1}{2} \tan ^{-1} \frac{U}{I_{l}-I_{r}} .
$$

The rule of composition of a number of independent plane-polarized streams can be formulated as follows: If we have a number of independent plane-polarized streams of intensities $I^{(n)}$ (say) in a given direction, the resulting mixture will be partially plane-polarized and will be described by the parameters

$$
I_{l}=\sum I^{(n)} \cos ^{2} \chi_{n} ; \quad I_{r}=\sum I^{(n)} \sin ^{2} \chi_{n},
$$

and

$$
U=\sum I^{(n)} \sin 2 \chi_{n}
$$

where $\chi_{n}$ denotes the inclination of the plane of polarization of the stream $I^{(n)}$ to the direction (in the transverse plane of the electric and the magnetic vectors) to which $I_{l}$ refers.

With the rule of composition, as we have just stated, the equations of transfer for $I_{l}, I_{r}$ and $U$ can be formulated in accordance, for example, with Rayleigh's laws. For the problem of diffuse reflection of a partially plane-polarized parallel beam of radiation (characterized by the parameters $F_{l}, F_{r}$ and $U^{(0)}$ ) incident on a plane-parallel atmosphere in the direction $\left(-\mu_{0}, \varphi_{0}\right)$, the three equations of transfer for the intensities $I_{l}, I_{r}$ and $U$ of the diffuse radiation field can be combined into a single vector equation of the form

$$
\begin{aligned}
\mu \frac{d I}{d \tau}=I(\tau, \mu, \varphi) & \\
& -\frac{3}{16 \pi} Q \int_{-1}^{+1} \int_{0}^{2 \pi} J\left(\mu, \varphi ;-\mu^{\prime}, \varphi^{\prime}\right) I\left(\tau, \mu^{\prime}, \varphi^{\prime}\right) d \mu^{\prime} d \varphi^{\prime} \\
& -\frac{3}{16} Q J\left(\mu, \varphi ; \mu_{0}, \varphi_{0}\right) F e^{-\tau / \mu_{0}},
\end{aligned}
$$

where

$$
\begin{gathered}
I=\left(I_{l}, I_{r}, U\right), \quad F=\left(F_{l}, F_{r}, U^{(0)}\right), \\
Q=\left(\begin{array}{lll}
1 & 0 & 0 \\
0 & 1 & 0 \\
0 & 0 & 2
\end{array}\right),
\end{gathered}
$$

and 
$J\left(\mu, \varphi ; \mu_{0}, \varphi_{0}\right)$

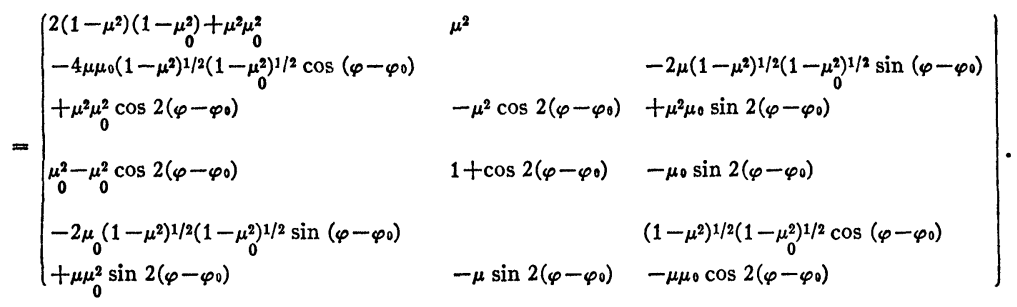

The matrix $Q J$ therefore plays the same role for this problem as the phase function $p(\cos \Theta)$ does in the simpler problems in which polarization is not allowed for.

We require to solve (VII) which satisfies the boundary condition

$$
I(0, \mu, \varphi) \equiv 0 \quad(-1<\mu<0) .
$$

Since the equation of transfer is linear in $F$, it is clear that the intensity $I\left(\mu, \varphi ; \mu_{0}, \varphi_{0}\right)$ diffusely reflected by the atmosphere in the outward direction $(\mu, \varphi)$ must be expressible in the form

$$
I\left(\mu, \varphi ; \mu_{0}, \varphi_{0}\right)=\frac{3}{16 \mu} \operatorname{QS}\left(\mu, \varphi ; \mu_{0}, \varphi_{0}\right) F .
$$

And our principal interest in this problem is in the determination of this scattering matrix $S$.

12. The Schwarzschild-Milne integral equation. In the preceding sections we have formulated several typical problems in the theory of radiative transfer and we have seen how all of them lead to integrodifferential equations of varying degrees of complexity. The question now arises as to whether there are any general methods for solving such equations. Until recently, the only example of such integrodifferential equations which had been considered to any extent is the simplest we have written down, namely equation (I). And here the method used was one of reducing the integro-differential equation to an integral equation. This reduction to an integral equation was achieved in the following way:

Writing equation (I) in the form

$$
\mu \frac{d I}{d \tau}=I-J(\tau),
$$

where

$$
J(\tau)=\frac{1}{2} \int_{-1}^{+1} I(\tau, \mu) d \mu,
$$


we observe that its formal solution is

$$
\begin{aligned}
I(\tau, \mu) & =\int_{\tau}^{\infty} e^{-(t-\tau) / \mu} J(t) \frac{d t}{\mu} & (0<\mu \leqq 1) \\
& =-\int_{0}^{\tau} e^{-(t-\tau) / \mu} J(t) \frac{d t}{\mu} & (-1 \leqq \mu<0) .
\end{aligned}
$$

Substituting this solution for $I(\tau, \mu)$ back into the equation defining $J(\tau)$ (equation (36)) we find, after some elementary reductions, that

$$
J(\tau)=\frac{1}{2} \int_{0}^{\infty} E_{1}(|t-\tau|) J(t) d t
$$

where

$$
E_{1}(x)=\int_{x}^{\infty} e^{-y} \frac{d y}{y}
$$

is the "first exponential integral." This is the Schwarzschild-Milne integral equation for $J(\tau)$.

13. The method of Hopf and Wiener. The integral equation (38) for $J(\tau)$ is seen to be homogeneous with a symmetric kernel. However, the kernel has a logarithmic singularity at $t=\tau$. Integral equations of this general structure have therefore a definite mathematical interest and have, in fact, been considered by a number of mathematicians including E. Hopf, J. Brönstein and N. Wiener. But this method based on the reduction of the equation of transfer to a linear integral equation for the source function has not so far been extended successfully to the solution of the other more interesting equations of transfer we have formulated. I shall therefore pass on to a different method of attack on these equations which has been developed during the past three years and which allows the solution of practically all the different types of transfer problems which arise in practice, by a systematic method of approximation. Also, for the particular problems enumerated, exact solutions for the angular distribution of the emergent (or the diffusely reflected) radiation can be found explicitly by passing to the limit of "infinite" approximation.

14. The method of replacing the integro-differential equations by systems of linear equations. The fundamental idea in this new method of solving an integro-differential equation is to replace it in a certain approximation by a system of ordinary linear equations. This reduction to an equivalent linear system is made by approximating the integrals over $\mu$ which occur in the equation by a weighted 
average of the values which the various integrands take at a suitably selected set of points in the interval $(-1,+1)$. It is evident that in making the necessary division of the interval $(-1,+1)$, we must be guided by reasons of "economy" in the sense that with a given number of divisions we must try to achieve the maximum accuracy in the evaluation of the integrals. The problem which we encounter here is therefore the same as that considered by Gauss in 1814 in deriving his formula for numerical quadratures. In this formula of Gauss, the interval $(-1,+1)$ is divided according to the zeros of a Legendre polynomial, $P_{m}(\mu)$ (say), and the integral of a function $f(\mu)$ over the interval $(-1,+1)$ is expressed as a sum in the form

$$
\int_{-1}^{+1} f(\mu) d \mu \simeq \sum a_{i} f\left(\mu_{j}\right)
$$

where the weights $a_{j}$ are given by

$$
a_{j}=\frac{1}{P_{m}^{\prime}\left(\mu_{j}\right)} \int_{-1}^{+1} \frac{P_{m}(\mu)}{\mu-\mu_{j}} d \mu .
$$

The reason Gauss's formula is superior to other formulae for quadratures in the interval $(-1,+1)$ is that for a given $m$ it evalues the integrals exactly for all polynomial of degree less than, or equal to, $2 m-1$; in other words, it is almost "twice as accurate" as we should expect a formula which uses only $m$ values of the function in the interval to be.

For certain formal reasons, we shall not enter into here, in our further work we shall use divisions of the interval $(-1,+1)$ only according to the zeros of the even Legendre polynomials. Further, when the division according to the zeros of $P_{2 n}(\mu)$ is selected, we shall say that we are working in the $n$th approximation. It is, therefore, apparent that when an equation of transfer is reduced to an equivalent linear system in the $n$th approximation, we are effectively using a polynomial representation of order $4 n-1$ (in $\mu$ ) for the various intensities which occur in our problem.

Finally, it may be observed that, when working in the $n$th approximation, we may use the following relations between the points of the Gaussian division, $\mu_{i}$, and the Gaussian weights $a_{j}$ :

$$
a_{i}=a_{-i} ; \quad \mu_{j}=-\mu_{-j} \quad(j= \pm 1, \cdots, \pm n),
$$

and

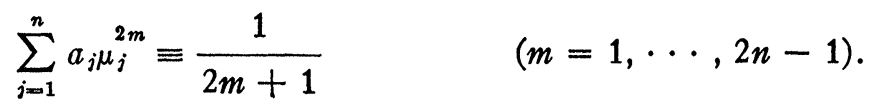


The identity (43) arises from the fact that in the $n$th approximation Gauss's formula evaluates exactly integrals of all polynomials of degree less than or equal to $4 n-1$.

15. The solution of equation (I) by the new method. We shall illustrate the method of solving linear integro-differential equations by reducing them to equivalent linear systems in the various approximations by considering equation $\mathrm{I}$.

In our $n$th approximation we replace equation (I) by the system of $2 n$ equations

$$
\mu_{i} \frac{d I_{i}}{d \tau}=I_{i}-\frac{1}{2} \sum a_{j} I_{j} \quad(i= \pm 1, \cdots, \pm n),
$$

where, for the sake of brevity, we have written $I_{i}(\tau)$ for $I\left(\tau, \mu_{i}\right)$.

In solving the system of equations represented by equation (44), we shall first obtain the different linearly independent solutions and later, by combining them, obtain the general solution.

As our first trial, we seek a solution of the form

$$
I_{i}=g_{i} e^{-k \tau} \quad(i= \pm 1, \cdots, \pm n),
$$

where the $g_{i}$ 's and $k$ are constants, for the present unspecified. Introducing equation (45) in equation (44), we obtain the relation

$$
g_{i}\left(1+\mu_{i} k\right)=\frac{1}{2} \sum a_{i} g_{j} .
$$

Hence,

$$
g_{i}=\frac{\text { constant }}{1+\mu_{i} k} \quad(i= \pm 1, \cdots, \pm n),
$$

where the "constant" is independent of $i$. Substituting the foregoing form for $g_{i}$ in equation (46), we obtain the characteristic equation for $k$ :

$$
1=\frac{1}{2} \sum \frac{a_{j}}{1+\mu_{j} k}
$$

Remembering that $a_{-i}=a_{j}$ and $\mu_{-j}=-\mu_{i}$, we can rewrite the characteristic equation in the form

$$
1=\sum_{j=1}^{n} \frac{a_{j}}{1-\mu_{j}^{2} k^{2}} .
$$

It is, therefore, seen that $k^{2}$ must satisfy an algebraic equation of order $n$. However, since 


$$
\sum_{j=1}^{n} a_{j}=1
$$

$k^{2}=0$ is a root of equation (49). Equation (48) accordingly admits only $2 n-2$ distinct nonzero roots which must occur in pairs as

$$
\pm k_{\alpha} \quad(\alpha=1, \cdots, n-1) .
$$

And corresponding to these $2 n-2$ roots, we have $2 n-2$ independent solutions of equation (44). The solution is completed by observing that

$$
I_{i}=b\left(\tau+\mu_{i}+Q\right) \quad(i= \pm 1, \cdots, \pm n),
$$

where $b$ and $Q$ are two arbitrary constants, also satisfies equation (44). The general solution of equation (44) can therefore be written in the form

$$
\begin{aligned}
& I_{i}=b\left\{\sum_{\alpha=1}^{n-1} \frac{L_{\alpha} e^{-k_{\alpha} \tau}}{1+\mu_{i} k_{\alpha}}+\sum_{\alpha=1}^{n-1} \frac{L_{-\alpha} e^{+k_{\alpha} \tau}}{1-\mu_{i} k_{\alpha}}+\tau+\mu_{i}+Q\right\} \\
& (i= \pm 1, \cdots, \pm n),
\end{aligned}
$$

where $b, L_{ \pm \alpha}(\alpha=1, \cdots, n-1)$ and $Q$ are the $2 n$ arbitrary constants of integration.

For the astrophysical problem on hand, the boundary conditions are that none of the $I_{i}$ 's increase exponentially as $\tau \rightarrow \infty$ and that, further, there is no radiation incident on $\tau=0$. The first of these conditions requires that in the general solution (53) we omit all terms in $\exp \left(+k_{\alpha} \tau\right)$, thus leaving

$$
I_{i}=b\left\{\sum_{\alpha=1}^{n-1} \frac{L_{\alpha} e^{-k_{\alpha \tau}}}{1+\mu_{i} k_{\alpha}}+\tau+\mu_{i}+Q\right\} \quad(i= \pm 1, \cdots, \pm n) .
$$

Next, the absence of any radiation in the directions $-1<\mu<0$ at $\tau=0$ implies that in our approximation we must require

$$
I_{-i}=0 \quad \text { at } \tau=0 \text { for } i=1, \cdots, n .
$$

Hence, according to equation (54),

$$
\sum_{\alpha=1}^{n-1} \frac{L_{\alpha}}{1-\mu_{i} k_{\alpha}}-\mu_{i}+Q=0 \quad(i=1, \cdots, n) .
$$

These are the $n$ equations which determine the $n$ constants of integration $L_{\alpha}(\alpha=1, \cdots, n-1)$ and $Q$. The constant $b$ is left arbitrary, and this, as we shall presently see, is related to the assigned constant net flux of radiation through the atmosphere. 
16. Some elementary identities. The further discussion of the solution obtained in $\$ 15$ requires certain relations which we shall now establish.

Let

$$
D_{m}(x)=\sum_{i} \frac{a_{i} \mu_{i}^{m}}{1+\mu_{i} x}=(-1)^{m} \sum_{i} \frac{a_{i} \mu_{i}^{m}}{1-\mu_{i} x} \quad(m=0, \cdots, 4 n) .
$$

There is a simple recursion formula which $D_{m}(x)$ defined in this manner satisfies. We have:

$$
D_{m}(x)=\frac{1}{x} \sum_{i} a_{i} \mu_{i}^{m-1}\left(1-\frac{1}{1+\mu_{i} x}\right),
$$

or, using equations (42) and (43),

$$
D_{m}(x)=\frac{1}{x}\left[\frac{2}{m} \epsilon_{m, \text { odd }}-D_{m-1}(x)\right] \text {, }
$$

where

$$
\boldsymbol{\epsilon}_{m, \text { odd }}= \begin{cases}1 & \text { if } m \text { is odd, } \\ 0 & \text { if } m \text { is even. }\end{cases}
$$

For odd, respectively, even values of $m$, equation (59) takes the form

$$
D_{2 j-1}(x)=\frac{1}{x}\left[\frac{2}{2 j-1}-D_{2 j-2}(x)\right],
$$

and

$$
D_{2 j}(x)=-\frac{1}{x} D_{2 j-1}(x) .
$$

Combining these relations, we have

$$
D_{2 j-1}(x)=\frac{1}{x}\left[\frac{2}{2 j-1}+\frac{1}{x} D_{2 j-3}(x)\right]=-x D_{2 j}(x) .
$$

From this formula, we readily deduce that

$$
\begin{aligned}
D_{2 j-1}(x)= & \frac{2}{(2 j-1) x}+\frac{2}{(2 j-3) x^{2}}+\cdots \\
& +\frac{2}{3 x^{2 j-3}}+\frac{2}{3 x^{2 j-1}}\left[2-D_{0}(x)\right]
\end{aligned}
$$

and 


$$
\begin{aligned}
D_{2 j}(x)= & -\frac{2}{(2 j-1) x^{2}}-\frac{2}{(2 j-3) x^{4}}-\cdots \\
& -\frac{2}{3 x^{2 j-2}}-\frac{2}{3 x^{2 j}}\left[2-D_{0}(x)\right] .
\end{aligned}
$$

If we now let $x$ be a root $k$ of the characteristic equation (48),

$$
D_{0}(k)=2 \text {, }
$$

and we find from equations (64) and (65) that

$$
\begin{gathered}
D_{1}(k)=D_{2}(k)=0, \\
D_{2 j}(k)=-\frac{2}{(2 j-1) k^{2}}-\frac{2}{(2 j-3) k^{4}}-\cdots-\frac{2}{3 k^{2 j-2}} \\
D_{2 j-1}(k)=\frac{2}{(2 j-1) k}+\frac{2}{(2 j-3) k^{3}}+\cdots+\frac{2}{3 k^{2 j-3}} \\
\quad(j=2, \cdots, n) .
\end{gathered}
$$

17. A relation between the characteristic roots and the zeros of the Legendre polynomial. In terms of the $D_{2 j}(k)$ 's introduced in $\$ 16$, we can express the characteristic equation for $k$ in a form which does not explicitly involve the Gaussian weights and divisions: Let $p_{2 j}$ be the coefficient of $\mu^{2 i}$ in the polynomial representation of the Legendre polynomial $P_{2 n}(\mu)$, so that

$$
P_{2 n}(\mu)=\sum_{j=0}^{n} p_{2 j} \mu^{2 j}
$$

Now consider

$$
\sum_{j=0}^{n} p_{2 j} D_{2 j}(k)=\sum_{i} \frac{a_{i}}{1+\mu_{i} k}\left(\sum_{j=0}^{n} p_{2 j} \mu_{i}^{2 j}\right) .
$$

Since the $\mu_{i}^{\prime}$ 's are the zeros of $P_{2 n}(\mu)$

$$
\sum_{j=0}^{n} p_{2 j}^{2 j} \equiv 0
$$

and the characteristic equation can be expressed in the form

$$
\sum_{j=0}^{n} p_{2 j} D_{2 j}(k)=0,
$$


where the $D_{2 j}$ 's are defined as in equation (68). Substituting in particular for $D_{2 n}$ and $D_{0}$, we have

$$
\frac{2}{3} \frac{p_{2 n}}{k^{2 n-2}}+\cdots+2 p_{0}=0 .
$$

From this equation it follows that

$$
\frac{1}{\left(k_{1} \cdots k_{n-1}\right)^{2}}=(-1)^{n-1} \frac{3 p_{0}}{p_{2 n}}=3 \mu_{1}^{2} \cdots \mu_{n}^{2},
$$

or

$$
k_{1} \cdots k_{n-1} \mu_{1} \cdots \mu_{n}=1 / 3^{1 / 2} .
$$

18. The law of darkening. Returning to the solution (54) of the transfer problem considered in $\$ 15$, we evaluate the net flux $\pi F$ according to the formula

$$
F=2 \int_{-1}^{+1} I \mu d \mu .
$$

In our present scheme of approximation, we can write

$$
F=2 \sum a_{i} I_{i} \mu_{i}
$$

Evaluating this sum with the $I_{i}$ 's given by equation (54), we obtain

$$
F=2 b\left\{\sum_{\alpha=1}^{n-1} L_{\alpha} e^{-k_{\alpha} \tau} D_{1}\left(k_{\alpha}\right)+\sum_{i} a_{i} \mu_{i}^{2}\right\} \text {. }
$$

Using equations (43) and (67), we have

$$
F=4 b / 3 \text {. }
$$

We can now rewrite the solution (54) in the form

$$
I_{i}=\frac{3}{4} F\left\{\sum_{\alpha=1}^{n-1} \frac{L_{\alpha} e^{-k_{\alpha} \tau}}{1+\mu_{i} k_{\alpha}}+\tau+\mu_{i}+Q\right\}(i= \pm 1, \cdots, \pm n)
$$

In terms of this solution, the source function for the problem under consideration can be obtained in the following manner. We have

$$
\begin{aligned}
J & =\frac{1}{2} \int_{-1}^{+1} I d \mu \simeq \frac{1}{2} \sum a_{j} I_{j} \\
& =\frac{3}{8} F\left\{\sum_{\alpha=1}^{n-1} L_{\alpha} e^{-k_{\alpha} \tau} D_{0}\left(k_{\alpha}\right)+2(\tau+Q)\right\},
\end{aligned}
$$


or, using equation (66),

$$
J=\frac{3}{4} F\left\{\sum_{\alpha=1}^{n-1} L_{\alpha} e^{-k_{\alpha} \tau}+\tau+Q\right\} .
$$

The angular distribution $I(0, \mu)$ of the emergent radiation can be found from the source function (83) in accordance with the formula (cf. equation (37))

$$
I(0, \mu)=\int_{0}^{\infty} J(\tau) e^{-\tau / \mu} \frac{d \tau}{\mu}
$$

We find

$$
I(0, \mu)=\frac{3}{4} F\left\{\sum_{\alpha=1}^{n-1} \frac{L_{\alpha}}{1+\mu k_{\alpha}}+\mu+Q\right\} .
$$

It is to be particularly noted that the foregoing expression for $I(0, \mu)$ is in agreement with the solution (81) for $\tau=0$ and at the points of the Gaussian division $\mu=\mu_{i}$.

Comparing equation (85) with equation (56) which determines the constants $L_{\alpha}$ and $Q$, we observe that the angular distribution of the emergent radiation defined for the interval $0 \leqq \mu \leqq 1$ is determined in terms of a function which has zeros assigned in the complementary interval $-1<\mu<0$. Thus, letting

$$
S(\mu)=\sum_{\alpha=1}^{n-1} \frac{L_{\alpha}}{1-\mu k_{\alpha}}-\mu+Q,
$$

the boundary conditions require that

$$
S\left(\mu_{i}\right)=0 \quad(i=1, \cdots, n),
$$

while the angular distribution of the emergent radiation is given by

$$
I(0, \mu)=\frac{3}{4} F S(-\mu) \text {. }
$$

19. The elimination of the constants and the expression of the solution in closed form. We shall now show how an explicit formula for $S(\mu)$ can be found without solving explicitly for the constants $L_{\alpha}$ and $Q$ :

Consider the function

$$
\prod_{\alpha=1}^{n-1}\left(1-\mu k_{\alpha}\right) S(\mu)
$$


This is a polynomial of degree $n$ in $\mu$ which vanishes for $\mu=\mu_{i}$, $i=1, \cdots, n$. Consequently there must exist a proportionality of the form

$$
\prod_{\alpha=1}^{n-1}\left(1-k_{\alpha} \mu\right) S(\mu) \propto \prod_{i=1}^{n-1}\left(\mu-\mu_{i}\right) .
$$

The constant of proportionality can be found from a comparison of the coefficients of the highest powers of $\mu$ on either side. In this manner we find that

$$
S(\mu)=(-1)^{n} k_{1} \cdots k_{n-1} \frac{\prod_{i=1}^{n}\left(\mu-\mu_{i}\right)}{\prod_{\alpha=1}^{n-1}\left(1-k_{\alpha} \mu\right)} .
$$

This is the required formula.

According to equation (91)

$$
S(-\mu)=k_{1} \cdots k_{n-1} \frac{\prod_{i=1}^{n}\left(\mu+\mu_{i}\right)}{\prod_{\alpha=1}^{n-1}\left(1+k_{\alpha} \mu\right)},
$$

or, using the result (76), we can write

$$
S(-\mu)=\frac{1}{3^{1 / 2}} H(\mu),
$$

where

$$
H(\mu)=\frac{1}{\mu_{1} \cdots \mu_{n}} \frac{\prod_{i=1}^{n}\left(\mu+\mu_{i}\right)}{\prod_{\alpha=1}^{n-1}\left(1+k_{\alpha} \mu\right)} .
$$

In terms of the function $H(\mu)$ defined in this manner, the angular distribution of the emergent radiation can be expressed in the form

$$
I(0, \mu)=\frac{3^{1 / 2}}{4} F H(\mu)
$$

20. The solution of equation (III) by the new method. A particular integral. As a further illustration of our method of solving equations 
of transfer, we shall next consider equation III appropriate for the problem of diffuse reflection by an isotropically scattering atmosphere. The equivalent system of linear equations in the $n$th approximation is

$$
\mu_{i} \frac{d I_{i}}{d \tau}=I_{i}-\frac{1}{2} \sum a_{j} I_{j}-\frac{1}{4} F e^{-\tau / \mu_{0}} \quad(i= \pm 1, \cdots, \pm n) .
$$

It is seen that the homogeneous system associated with equation (96) is the same as equation (44). Accordingly, the complimentary function for the solution of equation (96) is the same as the general solution (53) of the homogeneous system. To complete the solution we require only a particular integral. This can be found in the following manner: Setting

$$
I_{i}=\frac{1}{4} F h_{i} e^{-\tau / \mu_{0}} \quad(i= \pm 1, \cdots, \pm n)
$$

in equation (96) (the $h_{i}$ 's are constants unspecified for the present) we verify that we must have

$$
h_{i}\left(1+\mu_{i} / \mu_{0}\right)=\frac{1}{2} \sum a_{j} h_{j}+1 .
$$

Equation (98) implies that the constants $h_{i}$ must be expressible in the form

$$
h_{i}=\frac{\gamma}{1+\mu_{i} / \mu_{0}} \quad(i= \pm 1, \cdots, \pm n),
$$

where the constant $\gamma$ has to be determined from the condition (cf. equation (98))

$$
\gamma=\frac{1}{2} \gamma \sum \frac{a_{i}}{1+\mu_{i} / \mu_{0}}+1
$$

In other words

$$
I_{i}=\frac{1}{4} F \frac{\gamma e^{-\tau / \mu_{0}}}{1+\mu_{i} / \mu_{0}} \quad(i= \pm 1, \cdots, \pm n),
$$

with

$$
\gamma=\frac{1}{1-\sum_{j=1}^{n} \frac{a_{j}}{1-\mu_{j}^{2} / \mu_{0}^{2}}}
$$


represents the required particular integral of equation (96).

The constant $\gamma$ defined as in equation (102) can be expressed in terms of the $H$-function introduced in $\$ 19$ (equation (94)). For this purpose consider the function

$$
T(x)=1-\sum_{j=1}^{n} \frac{a_{j}}{1-\mu_{j}^{2} x} .
$$

This clearly vanishes for

$$
x=0 \quad \text { and } \quad x=k_{\alpha}^{2} \quad(\alpha=1, \cdots, n-1) .
$$

Accordingly

$$
T(x) \prod_{j=1}^{n}\left(1-{\left.\stackrel{2}{\mu_{j}} x\right)}^{2}\right)
$$

cannot differ from

$$
x \prod_{\alpha=1}^{n-1}\left(x-k_{\alpha}^{2}\right)
$$

by more than a constant factor since (105) represents a polynomial of degree $n$ in $x$. The constant of proportionality can be determined by comparing the coefficients of the highest powers. In this manner we find that

$$
T(x) \equiv(-1)^{n} \mu_{1}^{2} \cdots \mu_{n}^{2} \frac{x \prod_{\alpha=1}^{n-1}\left(x-k_{\alpha}^{2}\right)}{\prod_{j=1}^{n}\left(1-\mu_{i}^{2} x\right)}
$$

But

$$
\gamma=\frac{1}{T\left(\mu_{0}^{-2}\right)}
$$

Hence

$$
\gamma=(-1)^{n} \frac{1}{\mu_{1}^{2} \cdots \mu_{n}^{2}} \frac{\prod_{i=1}^{n}\left(\mu_{0}^{2}-\mu_{i}^{2}\right)}{\prod_{\alpha=1}^{n-1}\left(1-k_{\alpha}^{2} \mu_{0}^{2}\right)}
$$

or according to our definition of $H(\mu)$ 


$$
\gamma=H\left(\mu_{0}\right) H\left(-\mu_{0}\right) .
$$

21. The law of diffuse reflection for the case of isotropic scattering. Now, adding to the particular integral (101) the general solution of the homogeneous system compatible with our present requirement of boundedness of the solution for $\tau \rightarrow \infty$, we have

$$
\begin{array}{r}
I_{i}=\frac{1}{4} F\left\{\sum_{\alpha=1}^{n-1} \frac{L_{\alpha} e^{-k_{\alpha} \tau}}{1+\mu_{i} k_{\alpha}}+Q+\frac{H\left(\mu_{0}\right) H\left(-\mu_{0}\right)}{1+\mu_{i} / \mu_{0}} e^{-\tau / \mu_{0}}\right\} \\
(i=1, \cdots, n),
\end{array}
$$

where the constants $L_{\alpha}(\alpha=1, \cdots, n-1)$ and $Q$ are to be determined by the boundary conditions at $\tau=0$.

At $\tau=0$ we have no diffuse radiation directed inward. The conditions which determine the constants of integration are therefore

$$
\sum_{\alpha=1}^{n-1} \frac{L_{\alpha}}{1-\mu_{i} k_{\alpha}}+Q+\frac{H\left(\mu_{0}\right) H\left(-\mu_{0}\right)}{1-\mu_{i} / \mu_{0}}=0 \quad(i= \pm 1, \cdots, \pm n) .
$$

The angular distribution of the diffusely reflected radiation can be found in terms of the source function

$$
\Im(\tau)=\frac{1}{2} \sum a_{i} I_{i}+\frac{1}{4} F e^{-\tau / \mu_{0}},
$$

according to the formula

$$
I(0, \mu)=\int_{0}^{\infty} \Im(\tau) e^{-\tau / \mu} \frac{d \tau}{\mu} .
$$

We find

$$
I\left(0, \mu ; \mu_{0}\right)=\frac{1}{4} F\left\{\sum_{\alpha=1}^{n-1} \frac{L_{\alpha}}{1+\mu k_{\alpha}}+Q+\frac{H\left(\mu_{0}\right) H\left(-\mu_{0}\right)}{1+\mu / \mu_{0}}\right\} .
$$

As in $\$ 18$, we again observe that the angular distribution of the diffusely reflected radiation defined in the interval $0 \leqq \mu \leqq 1$ is described in terms of a function which has zeros assigned in the interval $0>\mu>-1$. Thus, letting

$$
S(\mu)=\sum_{\alpha=1}^{n-1} \frac{L_{\alpha}}{1-\mu k_{\alpha}}+Q+\frac{H\left(\mu_{0}\right) H\left(-\mu_{0}\right)}{1-\mu / \mu_{0}},
$$

we have

$$
S\left(\mu_{i}\right)=0 \quad(i=1, \cdots, n),
$$


and

$$
I\left(0, \mu ; \mu_{0}\right)=\frac{1}{4} F S(-\mu) .
$$

And, again as in the earlier problem, we can find an explicit formula for $S(\mu)$ without having to solve explicitly for the constants $L_{\alpha}$ and $Q$.

Thus, considering the function

$$
\left(1-\mu / \mu_{0}\right) \prod_{\alpha=1}^{n-1}\left(1-k_{\alpha} \mu\right) S(\mu)
$$

we observe that it is a polynomial of degree $n$ in $\mu$ which vanishes for $\mu=\mu_{i}, i=1, \cdots, n$. There must, therefore, exist a relation of the form

$$
S(\mu)=(-1)^{n} \frac{X}{\mu_{1} \cdots \mu_{n}} \frac{\prod_{i=1}^{n}\left(\mu-\mu_{i}\right)}{\prod_{\alpha=1}^{n-1}\left(1-k_{\alpha} \mu\right)} \frac{1}{\left(1-\mu / \mu_{0}\right)},
$$

where $X$ is a constant. In terms of $H(\mu)$ we can rewrite the foregoing equation as

$$
S(\mu)=X \frac{H(-\mu)}{1-\mu / \mu_{0}} .
$$

The constant $X$ appearing in equation (121) can be determined from the relation (cf. equation (116))

$$
\operatorname{limit}_{\mu \rightarrow \mu_{0}}\left(1-\mu / \mu_{0}\right) S(\mu)=H\left(\mu_{0}\right) H\left(-\mu_{0}\right)
$$

According to equation (121) the left-hand side of equation (122) is $X H\left(-\mu_{0}\right)$. Hence

$$
X=H\left(\mu_{0}\right)
$$

and

$$
S(\mu)=H\left(\mu_{0}\right) H(-\mu) \frac{\mu_{0}}{\mu_{0}-\mu} .
$$

The expression (118) for the angular distribution of the reflected radiation therefore becomes

$$
I\left(0, \mu ; \mu_{0}\right)=\frac{1}{4} F H(\mu) H\left(\mu_{0}\right) \frac{\mu_{0}}{\mu+\mu_{0}} .
$$


22. Tabulation of the solutions of equations (I)-(VII). The method of solution of transfer problems which we have illustrated in the preceding sections by considering the two simplest problems is, on examination, found to be sufficiently general for adaptation to the solution of the more difficult problems presented by the other equations of transfer formulated in $\$ \S 8-11$. While the details of the solution of these other equations (particularly equations VI and VII) are considerably more elaborate and complex, the analysis nevertheless shows similarities with the simple problems we have considered in the broad features. Thus, it is found that, in all cases, the angular distribution of the emergent (equivalently, reflected) radiation is described by a function for the argument in the range $(0,1)$ while the boundary conditions specify the zeros of the same function in the interval $(0,-1)$. This remarkable reciprocity, which exists in all the problems, enables the elimination of the constants and allows the reduction of the solutions to closed forms in the general $n$th approximation. And finally, these solutions, apart from certain constants, involve only $H$-functions of the form

$$
H(\mu)=\frac{1}{\mu_{1} \cdots \mu_{n}} \frac{\prod_{i=1}^{n}\left(\mu+\mu_{i}\right)}{\prod_{\alpha=1}^{n}\left(1+k_{\alpha} \mu\right)},
$$

where the $\mu_{i}$ 's are the zeros of the Legendre polynomial $P_{2 n}(\mu)$ and the $k_{\alpha}$ 's are the positive (or zero) roots of a characteristic equation of the form

$$
1=2 \sum_{j=1}^{n} \frac{a_{j} \Psi\left(\mu_{j}\right)}{1-k^{2} \mu_{j}^{2}},
$$

where $\Psi(\mu)$ is an even polynomial in $\mu$ satisfying the condition

$$
\int_{0}^{1} \Psi(\mu) d \mu \leqq \frac{1}{2} .
$$

(This last condition is necessary for $H(\mu)$ to be real.)

The different physical problems naturally lead to different characteristic equations and therefore to different $H$-functions. However, as the $H$-functions differ from one another only through the characteristic equations which define the roots $k_{\alpha}$, we may properly call $\Psi(\mu)$ the characteristic function in terms of which $H$ is defined.

We tabulate below the solutions for the various transfer problems 
(equations I-VII) obtained in the $n$th approximation of our method of solutions.

SOLUTIONS FOR THE EMERGENT AND THE DIFFUSELY REFLECTED RADIATION FOR VARIOUS TRANSFER PROBLEMS

\section{A. Isotropic scattering}

Problem with constant net flux: the law of darkening:

$$
I(\mu)=\frac{3^{1 / 2}}{4} F H(\mu) .
$$

Law of diffuse reflection:

$$
I\left(\mu ; \mu_{0}\right)=\frac{1}{4} F H(\mu) H\left(\mu_{0}\right) \frac{\mu_{0}}{\mu+\mu_{0}} .
$$

The characteristic function in terms of which $H(\mu)$ is defined is

$$
\Psi(\mu)=1 / 2 \text {. }
$$

B. Scattering in accordance with Rayleigh's phase function

$$
3\left(1+\cos ^{2} \Theta\right) / 4
$$

Problem with constant net flux: the law of darkening:

$$
I(\mu)=\frac{3}{4} q F H^{(0)}(\mu)
$$

Law of diffuse reflection:

$$
\begin{aligned}
I\left(\mu, \varphi ; \mu_{0}, \varphi_{0}\right) & =\frac{3}{32} F\left\{H^{(0)}(\mu) H^{(0)}\left(\mu_{0}\right)\left[3-\left(3-8 q^{2}\right)^{1 / 2}\left(\mu+\mu_{0}\right)+\mu_{0}\right]\right. \\
& -4 \mu \mu_{0}\left(1-\mu^{2}\right)^{1 / 2}\left(1-\mu_{0}^{2}\right)^{1 / 2} H^{(1)}(\mu) H^{(1)}\left(\mu_{0}\right) \cos \left(\varphi-\varphi_{0}\right) \\
& \left.+\left(1-\mu^{2}\right)\left(1-\mu_{0}^{2}\right) H^{(2)}(\mu) H^{(2)}\left(\mu_{0}\right) \cos 2\left(\varphi-\varphi_{0}\right)\right\} \frac{\mu_{0}}{\mu+\mu_{0}} .
\end{aligned}
$$

The characteristic functions in terms of which $H^{(0)}(\mu), H^{(1)}(\mu)$ and $H^{(2)}(\mu)$ are defined are respectively

$$
\begin{aligned}
& \Psi^{(0)}(\mu)=\frac{3}{16}\left(3-\mu^{2}\right), \\
& \Psi^{(1)}(\mu)=\frac{3}{8} \mu^{2}\left(1-\mu^{2}\right), \\
& \Psi^{(2)}(\mu)=\frac{3}{32}\left(1-\mu^{2}\right)^{2} .
\end{aligned}
$$


And finally,

$$
q=\frac{2\left(3^{1 / 2}\right)}{H^{(0)}\left(+3^{1 / 2}\right)-H^{(0)}\left(-3^{1 / 2}\right)}
$$

C. Scattering in accordance with the phase function $\lambda(1+x \cos \Theta)$

Law of diffuse reflection:

$I\left(\mu, \varphi ; \mu_{0}, \varphi_{0}\right)$

$$
\begin{aligned}
= & \frac{1}{4} \lambda F\left\{H^{(0)}(\mu) H^{(0)}\left(\mu_{0}\right)\left[1-c\left(\mu+\mu_{0}\right)-x(1-\lambda) \mu \mu_{0}\right]\right. \\
& \left.+x\left(1-\mu^{2}\right)^{1 / 2}\left(1-\mu_{0}^{2}\right)^{1 / 2} H^{(1)}(\mu) H^{(1)}\left(\mu_{0}\right) \cos \left(\varphi-\varphi_{0}\right)\right\} \frac{\mu_{0}}{\mu+\mu_{0}} .
\end{aligned}
$$

The characteristic functions in terms of which $H^{(0)}(\mu)$ and $H^{(1)}(\mu)$ are defined are respectively

$$
\begin{aligned}
& \Psi^{(0)}(\mu)=\frac{1}{2} \lambda\left[1+x(1-\lambda) \mu^{2}\right], \\
& \Psi^{(1)}(\mu)=\frac{1}{4} x \lambda\left(1-\mu^{2}\right) .
\end{aligned}
$$

The constant $c$ depends in a somewhat complicated manner on the characteristic roots defining $H^{(0)}(\mu)$ (cf. S. Chandrasekhar, Astrophysical Journal vol. 103 (1946) p. 165, equation [108]).

D. Scattering in accordance with Rayleigh's law and allowing for the polarization of the scattered radiation

Problem with constant net flux: the law of darkening in the two states of polarization:

$$
\begin{aligned}
& I_{l}(\mu)=\frac{3}{8} F\left(1-c^{2}\right)^{1 / 2} H_{l}(\mu), \\
& I_{r}(\mu)=\frac{3}{8} F \frac{1}{2^{1 / 2}} H_{r}(\mu)(\mu+c) .
\end{aligned}
$$

Problem of diffuse reflection: the scattering matrix:

$$
I\left(\mu, \varphi ; \mu_{0}, \varphi_{0}\right)=\frac{3}{16 \mu} Q S\left(\mu, \varphi ; \mu_{0}, \varphi_{0}\right) F
$$

where 

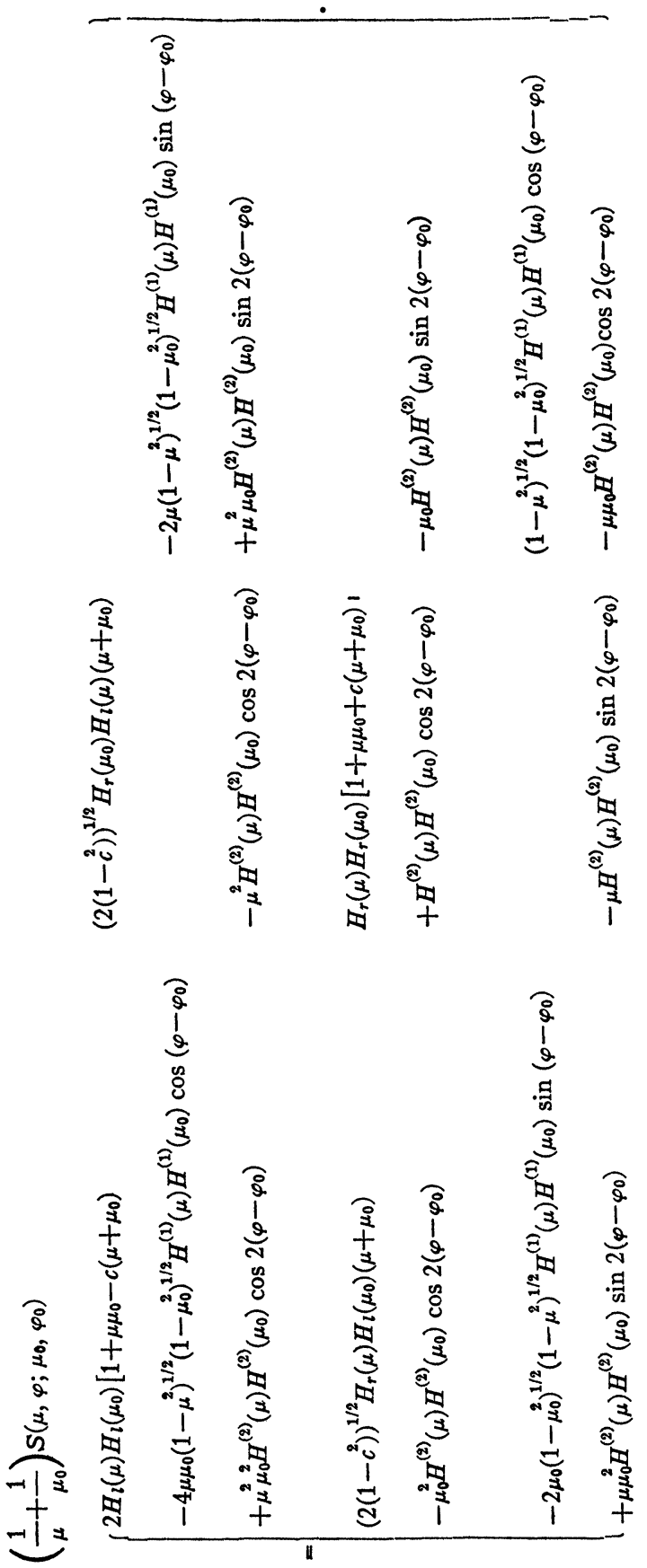
The characteristic functions in terms of which $H_{l}(\mu), H_{r}(\mu), H^{(1)}(\mu)$ and $H^{(2)}(\mu)$ are defined are respectively

$$
\begin{aligned}
\Psi_{l}(\mu) & =\frac{3}{4}\left(1-\mu^{2}\right) \\
\Psi^{(1)}(\mu) & =\frac{3}{8}\left(1-\mu^{2}\right)\left(1+2 \mu^{2}\right) \\
\Psi_{r}(\mu) & =\frac{3}{8}\left(1-\mu^{2}\right) \\
\Psi^{(2)}(\mu) & =\frac{3}{16}\left(1+\mu^{2}\right)^{2}
\end{aligned}
$$

And finally, the constant $c$ is given by

$$
c=\frac{H_{l}(+1) H_{r}(-1)+H_{l}(-1) H_{r}(+1)}{H_{l}(+1) H_{r}(-1)-H_{l}(-1) H_{r}(+1)} .
$$

23. Remarks on the tabulated solutions. The possibility of passage to the "infinite approximation." An examination of the solutions of the various transfer problems given in the preceding section discloses remarkable relationships between the laws of darkening of the emergent radiation from a semi-infinite plane-parallel atmosphere with constant net flux (and no incident radiation) and the laws of diffuse reflection by the same atmosphere. The relationship is naturally the simplest for the case of an isotropically scattering atmosphere and can indeed be established directly from the equations of transfer. However, in the other cases, the relationship is of a more complex nature and has to be sought between the darkening function for the problem with a constant net flux and the azimuth independent term in the law of diffuse reflection. It is seen that both these functions involve the same $H$-functions and the same constants.

While the relationship between the two problems has been established only in a particular scheme of approximation, it is apparent that the relationship itself must be an exact one since it is present in every approximation and must consequently be also present in the limit of infinite approximation when the solutions will become the exact ones of the problem. A further result of this train of thought is the realization that if we can solve the problem of diffuse reflection exactly, we shall, at the same time, have also solved exactly the axially symmetric problem with a constant net flux and no incident radiation. 
Having thus been led to conceive the passage to the limit of infinite approximation, we naturally ask ourselves: Can we in fact perform this limiting process and thus obtain the exact solutions for the various problems? The answer to this question must clearly depend on our ability to pass to the limit of the $H$-functions as we have defined them, as $n \rightarrow \infty$. We shall now indicate how this limiting process can be achieved in practice.

24. The equation satisfied by $H(\mu)$. For the purposes of passing to the limit of infinite approximations of the solutions of the various transfer problems, we shall first establish the following basic theorem relating to the $H$-functions.

THEOREM 1. Let $\Psi(\mu)$ be an even polynomial of degree $2 m$ in $\mu$ such that

$$
\int_{0}^{1} \Psi(\mu) d \mu \leqq \frac{1}{2} .
$$

Let $\mu_{j}(j= \pm 1, \cdots, \pm n)$ denote the division of the interval $(-1,+1)$ according to the zeros of the Legendre polynomial of order $2 n(>m)$; further, let $a_{j}\left(=a_{-j}\right)$ denote the corresponding Gaussian weights. Finally, let $k_{\alpha}(\alpha=1, \cdots, n)$ denote the distinct positive (or zero) roots of the characteristic equation

$$
1=\sum_{i} \frac{a_{j} \Psi\left(\mu_{j}\right)}{1+k \mu_{j}}=2 \sum_{j=1}^{n} \frac{a_{j} \Psi\left(\mu_{j}\right)}{1-k^{2} \mu^{2}} .
$$

Then the function

$$
H(\mu)=\frac{1}{\mu_{1} \cdots \mu_{n}} \frac{\prod_{i=1}^{n}\left(\mu+\mu_{i}\right)}{\prod_{\alpha=1}^{n}\left(1+k_{\alpha} \mu\right)}
$$

satisfies identically the equation

$$
H(\mu) \equiv 1+\mu H(\mu) \sum_{j=1}^{n} \frac{a_{j} H\left(\mu_{j}\right) \Psi\left(\mu_{j}\right)}{\mu+\mu_{j}} .
$$

Proof. We shall first consider the case

$$
\int_{0}^{1} \Psi(\mu) d \mu<\frac{1}{2}
$$


In this case the characteristic equation admits $n$ distinct nonvanishing positive roots and we consider the function

$$
S(\mu)=\sum_{\alpha=1}^{n} \frac{L_{\alpha}}{1-k_{\alpha} \mu}+1,
$$

where $L_{\alpha}(\alpha=1, \cdots, n)$ are certain constants to be determined from the equations

$$
\sum_{\alpha=1}^{n} \frac{L_{\alpha}}{1-k_{\alpha} \mu_{i}}+1=0 \quad(i=1, \cdots, n),
$$

or equivalently

$$
S\left(\mu_{i}\right)=0 \quad(i=1, \cdots, n) .
$$

By considerations of the type we are now familiar with, it can be shown that

$$
S(\mu)=k_{1} \cdots k_{n} \mu_{1} \cdots \mu_{n} \frac{(-1)^{n}}{\mu_{1} \cdots \mu_{n}} \frac{\prod_{i}\left(\mu-\mu_{i}\right)}{\prod_{\alpha}\left(1-k_{\alpha} \mu\right)} .
$$

In other words,

$$
S(\mu)=k_{1} \cdots k_{n} \mu_{1} \cdots \mu_{n} H(-\mu) .
$$

Since $H(0)=1$, we can rewrite equation (134) alternatively in the form

$$
S(\mu)=S(0) H(-\mu),
$$

where

$$
S(0)=\sum_{\alpha=1}^{n} L_{\alpha}+1=k_{1} \cdots k_{n} \mu_{1} \cdots \mu_{n} .
$$

Now, since $\Psi(\mu)$ is even in $\mu$, we can rewrite the equation which a characteristic root satisfies in either of the forms

$$
1=\sum_{i} \frac{a_{j} \Psi\left(\mu_{j}\right)}{1+k \mu_{i}}
$$

or

$$
1=\sum_{i} \frac{a_{j} \Psi\left(\mu_{j}\right)}{1-k \mu_{i}}
$$


Let $k_{\alpha}$ denote a particular characteristic root. Then, on account of equations (137) and (138), which are satisfied by any of the characteristic roots, we can clearly write

$$
\begin{aligned}
S(0)= & \sum_{\beta=1}^{n} L_{\beta}+1 \\
= & \sum_{\beta=1}^{n} \frac{L_{\beta}}{k_{\alpha}+k_{\beta}}\left[\sum_{j} a_{j} \Psi\left(\mu_{j}\right)\left\{\frac{k_{\alpha}}{1+k_{\alpha} \mu_{j}}+\frac{k_{\beta}}{1-k_{\beta} \mu_{j}}\right\}\right] \\
& +\sum_{i} \frac{a_{j} \Psi\left(\mu_{j}\right)}{1+k_{\alpha} \mu_{j}} .
\end{aligned}
$$

Simplifying the quantity in brackets in the foregoing equation, we have

$$
S(0)=\sum_{\beta=1}^{n} L_{\beta}\left[\sum_{j} \frac{a_{j} \Psi\left(\mu_{j}\right)}{\left(1+k_{\alpha} \mu_{j}\right)\left(1-k_{\beta} \mu_{j}\right)}\right]+\sum_{j} \frac{a_{j} \Psi\left(\mu_{j}\right)}{1+k_{\alpha} \mu_{j}}
$$

or, inverting the order of the summation,

$$
S(0)=\sum \frac{a_{j} \Psi\left(\mu_{j}\right)}{1+k_{\alpha} \mu_{j}}\left[\sum_{\beta=1}^{n} \frac{L_{\beta}}{1-k_{\beta} \mu_{j}}+1\right] .
$$

But the quantity in brackets in equation (141) is $S\left(\mu_{j}\right)$. Hence

$$
S(0)=\sum_{j} \frac{a_{j} S\left(\mu_{j}\right) \Psi\left(\mu_{j}\right)}{1+k_{\alpha} \mu_{j}} .
$$

In equation (142) (as in equations (137)-(141)) the summation is, of course, extended over all values of $j$, positive and negative. However, since $S\left(+\mu_{j}\right)=0$ (equation (132)), in equation (142) only the terms with negative $j$ make a nonzero contribution. We can, therefore, write

$$
S(0)=\sum_{j=1}^{n} \frac{a_{j} S\left(-\mu_{j}\right) \Psi\left(\mu_{j}\right)}{1-k_{\alpha} \mu_{j}} \quad(\alpha=1, \cdots, n)
$$

or, in view of equation (135),

$$
1=\sum_{j=1}^{n} \frac{a_{j} H\left(\mu_{j}\right) \Psi\left(\mu_{j}\right)}{1-k_{\alpha} \mu_{j}} \quad(\alpha=1, \cdots, n) .
$$

Now, consider the function

$$
1-\mu \sum_{j=1}^{n} \frac{a_{j} H\left(\mu_{j}\right) \Psi\left(\mu_{j}\right)}{\mu+\mu_{j}} .
$$


According to equation (144), this vanishes for $\mu=-1 / k_{\alpha} \quad(\alpha=1$, $\cdots, n)$; for

$$
1+\frac{1}{k_{\alpha}} \sum_{j=1}^{n} \frac{a_{j} H\left(\mu_{j}\right) \Psi\left(\mu_{j}\right)}{\left(-1 / k_{\alpha}\right)+\mu_{j}}=1-\sum_{j=1}^{n} \frac{a_{j} H\left(\mu_{j}\right) \Psi\left(\mu_{j}\right)}{1-k_{\alpha} \mu_{j}}=0 .
$$

Hence

$$
\prod_{j=1}^{n}\left(\mu+\mu_{j}\right)-\mu \sum_{j=1}^{n} a_{j} H\left(\mu_{j}\right) \Psi\left(\mu_{j}\right) \prod_{i \neq j}\left(\mu+\mu_{i}\right)
$$

also vanishes for

$$
\mu=-1 / k_{\alpha} \quad(\alpha=1, \cdots, n) .
$$

But the expression (147) is a polynomial of degree $n$ in $\mu$. It cannot, therefore, differ from

$$
\prod_{\alpha=1}^{n}\left(1+k_{\alpha} \mu\right)
$$

by more than a constant factor; and the constant of proportionality is seen to be

$$
\mu_{1} \cdots \mu_{n}
$$

from a comparison of the two functions at $\mu=0$. It therefore follows that

$$
1-\mu \sum_{j=1}^{n} \frac{a_{j} H\left(\mu_{j}\right) \Psi\left(\mu_{j}\right)}{\mu+\mu_{j}} \equiv \mu_{1} \cdots \mu_{n} \frac{\prod_{\alpha=1}^{n}\left(1+k_{\alpha} \mu\right)}{\prod_{j=1}^{n}\left(\mu+\mu_{j}\right)}=\frac{1}{H(\mu)}
$$

Hence

$$
H(\mu) \equiv 1+\mu H(\mu) \sum_{j=1}^{n} \frac{a_{j} H\left(\mu_{i}\right) \Psi\left(\mu_{j}\right)}{\mu+\mu_{i}} .
$$

This proves the theorem for the case (129).

Turning now to the case

$$
\int_{0}^{1} \Psi(\mu) d \mu=\frac{1}{2}
$$

we observe that, in this case, $k=0$ is a root of the characteristic equation, and we are left with only $(n-1)$ positive roots. We, therefore, 
consider in this case the function

$$
S(\mu)=\sum_{\alpha=1}^{n-1} \frac{L_{\alpha}}{1-k_{\alpha} \mu}+L_{0}-
$$

in place of equation (130). However, the constants $L_{0}$ and $L_{\alpha}$ $(\alpha=1, \cdots, n-1)$ are again to be determined by the conditions

$$
S\left(\mu_{i}\right)=0 \quad(i=1, \cdots, n) .
$$

With this definition of $S(\mu)$, equation (135) continues to be valid and the rest of the proof follows on similar lines. The only essential point of departure that needs to be noted is that, at the stage of the proof corresponding to equation (140) and before inverting the order of the summation, we must add the extra term

$$
-\sum_{j} \frac{a_{j} \Psi\left(\mu_{j}\right) \mu_{j}}{1+k_{\alpha} \mu_{j}}
$$

to the right-hand side of the equation. We can do this without altering anything, since the quantity we thus add is zero; for

$$
\begin{aligned}
\sum_{j} \frac{a_{j} \Psi\left(\mu_{j}\right) \mu_{j}}{1+k_{\alpha} \mu_{j}} & =\frac{1}{k_{\alpha}}\left[\sum_{i} a_{j} \Psi\left(\mu_{j}\right)\left\{1-\frac{1}{1+k_{\alpha} \mu_{j}}\right\}\right] \\
& =\frac{1}{k_{\alpha}}\left[1-\sum_{j} \frac{a_{j} \Psi\left(\mu_{j}\right)}{1+k_{\alpha} \mu_{j}}\right]=0 .
\end{aligned}
$$

(Note that we are permitted to set $\sum a_{j} \Psi\left(\mu_{j}\right)=1$, since the Gauss sum in the $n$th approximation evaluates the integrals exactly for all polynomials of degree less than or equal to $4 n-1$; and we have assumed $2 n>m$.)

This completes the proof of the theorem.

25. The limit of $H(\mu)$ as $n \rightarrow \infty$. The basic functional equation. The theorem proved in $\$ 24$ suggests how the limit of the $H$-function as $n \rightarrow \infty$ can be obtained. We shall state this in the form of a theorem.

THEOREM 2. The solution of the functional equation

$$
H(\mu)=1+\mu H(\mu) \int_{0}^{1} \frac{H\left(\mu^{\prime}\right) \Psi\left(\mu^{\prime}\right)}{\mu+\mu^{\prime}} d \mu^{\prime},
$$

where $\Psi(\mu)$ is an even polynomial satisfying the condition

$$
\int_{0}^{1} \Psi(\mu) d \mu \leqq \frac{1}{2}
$$


is the limit function

$$
\operatorname{limit}_{n \rightarrow \infty} \frac{1}{\mu_{1} \cdots \mu_{n}} \frac{\prod_{i=1}^{n}\left(\mu+\mu_{i}\right)}{\prod_{\alpha=1}^{n}\left(1+k_{\alpha} \mu\right)}
$$

where the $\mu_{i}$ 's and the $k_{\alpha}$ 's have the same meanings as in Theorem 1.

Sketch of PROOF. The theorem arises in the following way: It is known that the integral of a bounded function over the interval $(0,1)$ can be approximated by a Gauss sum with any desired degree of accuracy by choosing a division of the interval according to the zeros of a Legendre polynomial of a sufficiently high degree. The integral which occurs on the right-hand side of the functional equation for $H(\mu)$ can, therefore, be replaced by the Gauss sum

$$
\sum_{j=1}^{n} \frac{a_{j} H\left(\mu_{j}\right) \Psi\left(\mu_{j}\right)}{\mu+\mu_{j}}
$$

to any desired accuracy by choosing a sufficiently large $n$. But by Theorem 1 , for a finite $n$, no matter how large, the unique solution of the equation

$$
H(\mu)=1+\mu H(\mu) \sum_{j=1}^{n} \frac{a_{j} H\left(\mu_{j}\right) \Psi\left(\mu_{j}\right)}{\mu+\mu_{j}}
$$

is

$$
\frac{1}{\mu_{1} \cdots \mu_{n}} \frac{\prod_{i=1}^{n}\left(\mu+\mu_{i}\right)}{\prod_{\alpha=1}^{n}\left(1+k_{\alpha} \mu\right)} .
$$

If we now let $n \rightarrow \infty$, equation (159) becomes

$$
H(\mu)=1+\mu H(\mu) \int_{0}^{1} \frac{H\left(\mu^{\prime}\right) \Psi\left(\mu^{\prime}\right)}{\mu+\mu^{\prime}} d \mu^{\prime} .
$$

The solution of this functional equation is, therefore, seen to be the limit of the function (160) as $n \rightarrow \infty$.

It is realized that the sketch of the proof of Theorem 2 we have just outlined does not meet the full demands of a rigorous mathematical demonstration. It is, moreover, probable that precisely the 
questions of uniqueness and existence which we have ignored will cause the principal difficulties in the constructions of a rigorous mathematical proof. However, as the equation arises in a physical context, and the physical situations are such as to leave no room for ambiguity, it is hardly to be doubted that the theorem is true. Indeed, as we shall presently show quite rigorously by an entirely different line of argument, the exact solutions for the various transfer problems are of the forms tabulated in $\$ 22$ with the $H$-functions redefined in terms of functional equations of the form (161) instead of in terms of the Gaussian division and characteristic roots. Nevertheless, it may be of interest to pursue further the purely mathematical questions raised by Theorem 2 .

26. A practical method of determining the exact $H$-functions as solutions of the functional equations they satisfy. Assuming for the present the indications of the preceding sections that the exact solutions for the various transfer problems are of the forms found in our method of solution and that the $H$-functions which occur in them have to be redefined as solutions of functional equations of the form (161), we may observe that Theorem 1 of $\$ 24$ suggests a simple practical method for determining the exact $H$-functions numerically. For, starting with an approximate solution for $H(\mu)$ (in the third approximation, for example) we can determine the exact $H$-functions by a process of iteration using for this purpose the functional equation which it satisfies. In this manner the exact $H$-functions which occur in the solutions of the various transfer problems involving isotropic scattering with an albedo $\lambda \leqq 1$, Rayleigh phase function and Rayleigh scattering (including the state of polarization of the scattered radiation) have all been numerically evaluated. We have therefore now available exact numerical solutions for the cases A, B, C (for $x=0$ ) and D tabulated in $\$ 22$.

27. The constants in the solution. It is to be noted that the determination of the limit to which the $H$-functions which occur in the solutions for the emergent (or diffusely reflected) radiation in the $n$th approximation tend, as $n \rightarrow \infty$, still leaves open the question of the exact limiting values of the constants which occur in these solutions. It does not seem that any direct or simple limiting process can be applied to the formulae which define them in the $n$th approximation. Attention may be particularly drawn in this connection to the fact that the formulae which define these constants (in the $n$th approximation) often involve the values of $H$-functions (now defined as rational functions in terms of the Gaussian division and the characteristic 
roots) outside the interval $(0,1)$ (sometimes even for complex values of the argument!), whereas it would seem that in the limit of infinite approximation $H(\mu)$ has a meaning only in the interval $(0,1)$. (See, for example, the definitions of the constants $c$ and $q$ under the headings $B$ and D in the tabulation of \$22.) However, it appears that these constants can be determined indirectly by appealing to certain other identical relations which the problems must satisfy.

Thus, considering the transfer problems involving the Rayleigh phase function, we have

$$
I(\mu)=\frac{3}{4} q F H^{(0)}(\mu),
$$

where $H^{(0)}(\mu)$ is defined as the solution of the functional equation

$$
H^{(0)}(\mu)=1+\frac{3}{16} \mu H^{(0)}(\mu) \int_{0}^{1} \frac{\left(3-\mu^{\prime 2}\right) H\left(\mu^{\prime}\right)}{\mu+\mu^{\prime}} d \mu^{\prime} .
$$

Now, as equation (162) gives the angular distribution of the emergent radiation for the axially symmetric problem with constant net flux, it follows that the outward flux of the emergent radiation must also equal $\pi F$. In other words, we must have

$$
F=2 \int_{0}^{1} I(\mu) \mu d \mu=\frac{3}{2} q F \alpha_{1}
$$

where $\alpha_{1}$ denotes the first moment of $H^{(0)}(\mu)$. Hence,

$$
q=\frac{2}{3 \alpha_{1}} .
$$

Thus, once the solution of equation (163) has been determined (by iteration based on an approximate $H(\mu)$ as suggested in \$26) the constant $q$ can be determined directly in terms of its first moment. Since $q$ is the only constant which occurs in the solutions, they become determinate in this fashion.

Similarly, in the transfer problems in which the polarization of the scattered radiation in accordance with Rayleigh's law has to be properly allowed for, the solutions again involve a constant $c$. It does not seem possible to pass directly to the limit of infinite approximation in the formula defining this constant in the $n$th approximation. But we can determine it by appealing to the flux condition in the problem with the constant net flux. Thus, with the solutions $I_{l}(\mu)$ and $I_{r}(\mu)$ for the emergent radiations in the two states of polarization as given in $\$ 22$ (with the functions $H_{l}(\mu)$ and $H_{r}(\mu)$ now defined properly in 
terms of functional equations of the form (161)), we must have

$$
\begin{aligned}
F & =2 \int_{0}^{1}\left[I_{l}(\mu)+I_{r}(\mu)\right] \mu d \mu \\
& =\frac{3}{4} F\left[\left(1-c^{2}\right)^{1 / 2} \alpha_{1}+\frac{1}{2^{1 / 2}}\left(A_{2}+c A_{1}\right)\right]
\end{aligned}
$$

where $\alpha_{1}$ denotes the first moment of $H_{l}(\mu)$ and $A_{1}$ and $A_{2}$ are the first and the second moments, respectively, of $H_{r}(\mu)$. Hence, we can determine $c$ from the equation

$$
\left(2\left(1-c^{2}\right)\right)^{1 / 2} \alpha_{1}+A_{2}+c A_{1}=\frac{4}{3} 2^{1 / 2} .
$$

28. The functional equation for the problem of diffuse reflection. The discussion in the preceding sections has shown how we can obtain in practice the exact solutions for the angular distribution of the emergent and the reflected radiations from a semi-infinite planeparallel atmosphere for a wide variety of scattering laws. From a strictly mathematical point of view, the limiting process by which the passage to the limit of infinite approximation was achieved may not have been as rigorously justified as one might have wished. We shall, therefore, now show how the exact solutions obtained in the manner of the preceding sections (by redefining, for example, the $H$-functions which occur in the solutions in the general $n$th approximation, in terms of functional equations of the form (161)) can be justified by following an entirely different line of argument. The basic idea in the development we are now going to describe is due to the Armenian astrophysicist, V. A. Ambarzumian.

In the problem of diffuse reflection, we are interested in the solution of the relevant equations of transfer principally, only to the extent that we want to establish the law of diffuse reflection as specified by the $\sigma\left(\mu, \varphi ; \mu_{0}, \varphi_{0}\right)$ which gives the intensity reflected in the direction $(\mu, \varphi)$ when a parallel beam of radiation of unit flux normal to itself is incident on the atmosphere in the direction $\left(-\mu_{0}, \varphi_{0}\right)$. Now, Ambarzumian starts with the almost trivial observation that the intensity $\sigma\left(\mu, \varphi ; \mu_{0}, \varphi_{0}\right)$ must be invariant to the addition (or removal) of layers of arbitrary optical thickness to (from) the atmosphere and shows (and this is really the point of the observation) how this invariance can be used to derive a functional equation for the scattering function $\sigma\left(\mu, \varphi ; \mu_{0}, \varphi_{0}\right) \mu$. Ambarzumian has explicitly derived the form of this functional equation for the law of diffuse reflection 
from an atmosphere scattering radiation in accordance with a general phase function, that is, the functional equation associated with the equation of transfer (24). However, when one proceeds to solve the resulting functional equation, one is soon led to simultaneous systems of nonlinear, nonhomogeneous functional equations of such a highly complex nature that one might almost despair of solving them! But, on examination, it soon appears that a knowledge of the forms of the solution obtained by the method described in the earlier parts of this lecture enables us, in all cases, to reduce the Ambarzumian type of functional equations to equations of the following standard form

$$
H(\mu)=1+\mu H(\mu) \int_{0}^{1} \frac{B\left(\mu^{\prime}\right) \Psi\left(\mu^{\prime}\right)}{\mu+\mu^{\prime}} d \mu^{\prime},
$$

and helps us, moreover, to confirm the results obtained by our method of passage to the limit of infinite approximation.

29. The functional equation for the scattering matrix. In this section we shall derive, following Ambarzumian's general ideas, the functional equation for the scattering matrix $\boldsymbol{S}$ introduced in $\$ 11$ (equation (34)). This problem is, therefore, more advanced than the ones conered by Ambarzumian; but it serves to illustrate the power of his idea.

To obtain the functional equation for $S$, we first rewrite the equation of transfer VII in the form

$$
\mu \frac{d I}{d \tau}=I(\tau, \mu, \varphi)-B(\tau, \mu, \varphi),
$$

where

$$
\begin{aligned}
B(\tau, \mu, \varphi)= & \frac{3}{16 \pi} Q \int_{-1}^{+1} \int_{0}^{2 \pi} J\left(\mu, \varphi ;-\mu^{\prime}, \varphi^{\prime}\right) I\left(\tau, \mu^{\prime}, \varphi^{\prime}\right) d \mu^{\prime} d \varphi^{\prime} \\
& +\frac{3}{16} Q J\left(\mu, \varphi ; \mu_{0}, \varphi_{0}\right) F e^{-\tau / \mu_{0}},
\end{aligned}
$$

and express the intensity $I\left(\mu, \varphi ; \mu_{0}, \varphi_{0}\right)$ reflected in the direction $(\mu, \varphi)$, where radiation with a net flux $\pi F$ is incident in the direction $\left(-\mu_{0}, \varphi_{0}\right)$, in the form

$$
I\left(\mu, \varphi ; \mu_{0}, \varphi_{0}\right)=\frac{3}{16 \mu} Q S\left(\mu, \varphi ; \mu_{0}, \varphi_{0}\right) F
$$

Now, consider a level at a depth $d \tau$ below the boundary of the 
atmosphere at $\tau=0$. At this level the radiation field present can be decomposed into two parts: first, there is the reduced incident flux of amount

$$
\pi F\left(1-\frac{d \tau}{\mu_{0}}\right)
$$

and, second, there is a diffuse radiation field. The amount of this diffuse radiation field which is directed inward can be inferred from the equation of transfer: for, since at $\tau=0$ there is no inward intensity, at the level $d \tau$, we must have an inward intensity

$$
I\left(d \tau,-\mu^{\prime}, \varphi^{\prime}\right)=B\left(0,-\mu^{\prime}, \varphi^{\prime}\right) \frac{d \tau}{\mu^{\prime}}
$$

in the direction $\left(-\mu^{\prime}, \varphi^{\prime}\right)$. Both of these radiation fields will be reflected by the atmosphere below $d \tau$ by the same laws as those by which the atmosphere below $\tau=0$ reflects. This invariance is $d u e$ to the fact that the removal of a layer of arbitrary thickness from a semi-infinite atmosphere cannot alter its reflecting power. This is Ambarzumian's basic idea. Accordingly, the reflection of the radiations (172) and (173) by the atmosphere below $d \tau$ will contribute to an outward intensity, in the direction $(\mu, \varphi)$, the amount

$$
\begin{aligned}
I(d \tau, \mu, \varphi) & =\frac{3}{16 \mu}\left(1-\frac{d \tau}{\mu_{0}}\right) Q S\left(\mu, \varphi ; \mu_{0}, \varphi_{0}\right) F \\
+ & \frac{3}{16 \pi \mu} d \tau Q \int_{0}^{1} \int_{0}^{2 \pi} S\left(\mu, \varphi ; \mu^{\prime}, \varphi^{\prime}\right) B\left(0,-\mu^{\prime}, \varphi^{\prime}\right) \frac{d \mu}{\mu^{\prime}} d \varphi^{\prime} .
\end{aligned}
$$

On the other hand, from the equation of transfer, we conclude that

$$
\begin{aligned}
I(d \tau, \mu, \varphi) & =I(0, \mu, \varphi)+\frac{d \tau}{\mu}[I(0, \mu, \varphi)-B(0, \mu, \varphi)] \\
& =\frac{3}{16 \mu}\left(1+\frac{d \tau}{\mu}\right) \operatorname{SS}\left(\mu, \varphi ; \mu_{0}, \varphi_{0}\right) F-\frac{d \tau}{\mu} B(0, \mu, \varphi) .
\end{aligned}
$$

Combining equations (174) and (175) and passing to the limit $d \tau=0$, we have

$$
\begin{aligned}
& \frac{3}{16}\left(\frac{1}{\mu}+\frac{1}{\mu_{0}}\right) Q S\left(\mu, \varphi ; \mu_{0}, \varphi_{0}\right) F=B(0, \mu, \varphi) \\
& \quad+\frac{3}{16 \pi} Q \int_{0}^{1} \int_{0}^{2 \pi} S\left(\mu, \varphi ; \mu^{\prime}, \varphi^{\prime}\right) B\left(0,-\mu^{\prime}, \varphi^{\prime}\right) \frac{d \mu^{\prime}}{\mu^{\prime}} d \varphi^{\prime} .
\end{aligned}
$$


But, according to equations (170) and (171)

$$
\begin{aligned}
& B(0, \mu, \varphi)=\frac{3}{16} Q\left[J\left(\mu, \varphi ; \mu_{0}, \varphi_{0}\right)\right. \\
& \left.+\frac{3}{16 \pi} \int_{0}^{1} \int_{0}^{2 \pi} J\left(\mu, \varphi ;-\mu^{\prime \prime}, \varphi^{\prime \prime}\right) Q S\left(\mu^{\prime \prime}, \varphi^{\prime \prime} ; \mu_{0}, \varphi_{0}\right) \frac{d \mu^{\prime \prime}}{\mu^{\prime \prime}} d \varphi^{\prime \prime}\right] F .
\end{aligned}
$$

Substituting for $B(0, \mu, \varphi)$ from the foregoing equation in equation (176) and remembering that $F$ can be an arbitrary vector, we find, after some minor reductions, that

$$
\begin{aligned}
& \left(\frac{1}{\mu}+\frac{1}{\mu_{0}}\right) S\left(\mu, \varphi ; \mu_{0}, \varphi_{0}\right)=J\left(\mu, \varphi ; \mu_{0}, \varphi_{0}\right) \\
& +\frac{3}{16 \pi} \int_{0}^{1} \int_{0}^{2 \pi} J\left(\mu, \varphi ;-\mu^{\prime \prime}, \varphi^{\prime \prime}\right) Q S\left(\mu^{\prime \prime}, \varphi^{\prime \prime} ; \mu_{0}, \varphi_{0}\right) \frac{d \mu^{\prime \prime}}{\mu^{\prime \prime}} d \varphi^{\prime \prime} \\
& +\frac{3}{16 \pi} \int_{0}^{1} \int_{0}^{2 \pi} S\left(\mu, \varphi ; \mu^{\prime}, \varphi^{\prime}\right) Q J\left(-\mu^{\prime}, \varphi^{\prime} ; \mu_{0}, \varphi_{0}\right) \frac{d \mu^{\prime}}{\mu^{\prime}} d \varphi^{\prime} \\
& +\frac{9}{256 \pi^{2}} \int_{0}^{1} \int_{0}^{2 \pi} \int_{0}^{1} \int_{0}^{2 \pi} S\left(\mu, \varphi ; \mu^{\prime}, \varphi^{\prime}\right) Q J\left(-\mu^{\prime}, \varphi^{\prime} ;-\mu^{\prime \prime}, \varphi^{\prime \prime}\right) Q \\
& \times S\left(\mu^{\prime \prime}, \varphi^{\prime \prime} ; \mu_{0}, \varphi_{0}\right) \frac{d \mu^{\prime}}{\mu^{\prime}} d \varphi^{\prime} \frac{d \mu^{\prime \prime}}{\mu^{\prime \prime}} d \varphi^{\prime \prime} .
\end{aligned}
$$

This is the required functional equation for the scattering matrix.

Now, the matrix $J$ (see equation (32)) has the property

$$
J_{i k}\left(\mu, \varphi ; \mu_{0}, \varphi_{0}\right)=J_{k i}\left(\mu_{0}, \varphi ; \mu, \varphi_{0}\right) .
$$

From this property of $J$ for transposition, it follows from equation (178) that $S$ has also the same property:

$$
S_{i k}\left(\mu, \varphi ; \mu_{0}, \varphi_{0}\right)=S_{k i}\left(\mu_{0}, \varphi ; \mu, \varphi_{0}\right) .
$$

It can be verified that equation (180) is equivalent to Helmholtz' principle of reciprocity for the problem under consideration.

30. The reduction of the functional equation for $S$. We shall now indicate how the functional equation for $\boldsymbol{S}$ derived in the preceding section can be solved.

From the form of the equation for $S$ and the manner of its relation to $J$, it is evident that in a Fourier analysis of the elements of $S$ in $\left(\varphi-\varphi_{0}\right)$ we must have the same nonvanishing components as in the corresponding elements of $J$. We may accordingly assume without loss of generality that $S$ has the form 


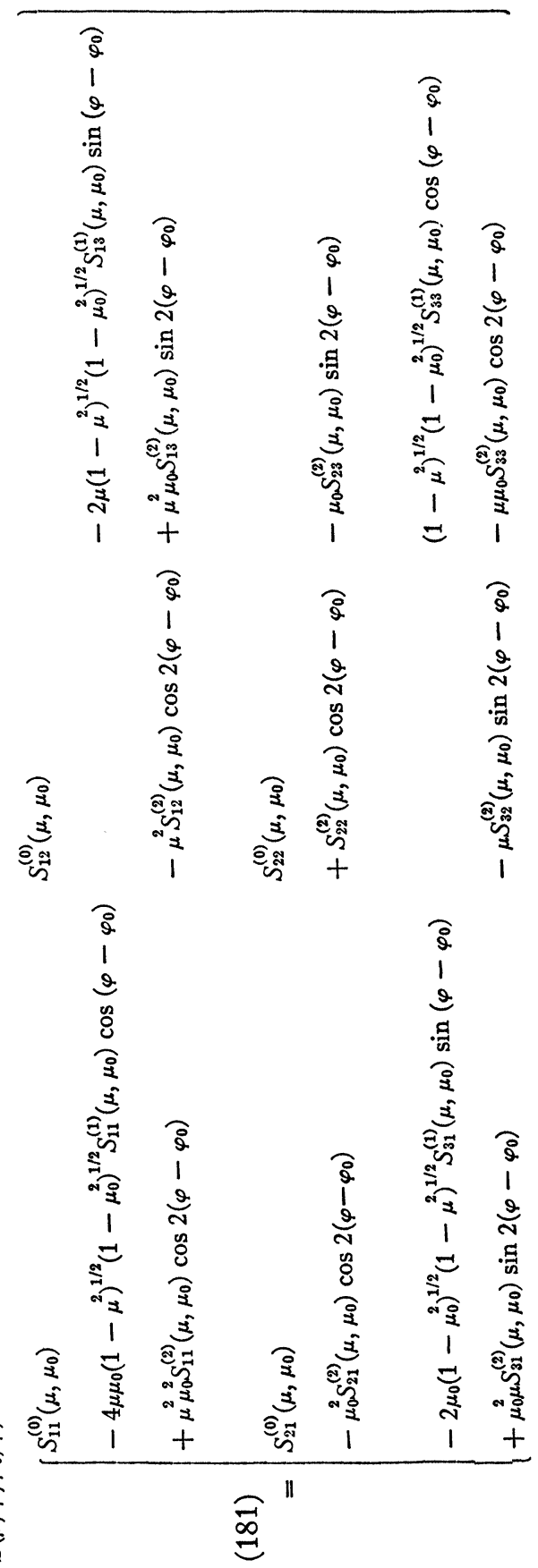


where, as the notation indicates, $S_{11}^{(0)}$, and so on, are all functions of $\mu$ and $\mu_{0}$ only. From the property (180) of $S$ for transposition, we now conclude that

$$
S_{j k}^{(i)}\left(\mu, \mu_{0}\right)=S_{k j}^{(i)}\left(\mu_{0}, \mu\right) .
$$

If we now substitute the form (181) for $S$ in equation (178) and equate the different Fourier components of the various elements, we shall clearly obtain three systems of functional equations governing the functions of the different orders, distinguished by their superscripts. Of these systems, the first, involving the zero-order functions $S_{11}^{(0)}, S_{12}^{(0)}, S_{21}^{(0)}$ and $S_{22}^{(0)}$, is the most important and, at the same time, the most difficult. We shall accordingly consider this system briefly.

First, we may write down the equations which are found for $S_{11}^{(0)}$, $S_{12}^{(0)}, S_{21}^{(0)}$, and $S_{22}^{(0)}$. The equations are

$$
\begin{aligned}
& \left(\frac{1}{\mu}+\frac{1}{\mu_{0}}\right) S_{11}^{(0)}\left(\mu, \mu_{0}\right) \\
& =\left\{\mu^{2}+\frac{3}{8} \int_{0}^{1} \frac{d \mu^{\prime}}{\mu^{\prime}}\left[\mu^{\prime 2} S_{11}^{(0)}\left(\mu, \mu^{\prime}\right)+S_{12}^{(0)}\left(\mu, \mu^{\prime}\right)\right]\right\} \\
& \times\left\{\mu_{0}^{2}+\frac{3}{8} \int_{0}^{1} \frac{d \mu^{\prime}}{\mu^{\prime}}\left[\mu^{\prime 2} S_{11}^{(0)}\left(\mu^{\prime}, \mu_{0}\right)+S_{21}^{(0)}\left(\mu^{\prime}, \mu_{0}\right)\right]\right\} \\
& +2\left\{1-\mu^{2}+\frac{3}{8} \int_{0}^{1} \frac{d \mu^{\prime}}{\mu^{\prime}}\left(1-\mu^{\prime 2}\right) S_{11}^{(0)}\left(\mu, \mu^{\prime}\right)\right\} \\
& \times\left\{1-\mu_{0}^{2}+\frac{3}{8} \int_{0}^{1} \frac{d \mu^{\prime}}{\mu^{\prime}}\left(1-\mu^{\prime 2}\right) S_{11}^{(0)}\left(\mu^{\prime}, \mu_{0}\right)\right\} \text {; } \\
& \left(\frac{1}{\mu}+\frac{1}{\mu_{0}}\right) S_{12}^{(0)}\left(\mu, \mu_{0}\right) \\
& =\left\{\mu^{2}+\frac{3}{8} \int_{0}^{1} \frac{d \mu^{\prime}}{\mu^{\prime}}\left[\mu^{\prime 2} S_{11}^{(0)}\left(\mu, \mu^{\prime}\right)+S_{12}^{(0)}\left(\mu, \mu^{\prime}\right)\right]\right\} \\
& \times\left\{1+\frac{3}{8} \int_{0}^{1} \frac{d \mu^{\prime}}{\mu^{\prime}}\left[\mu^{\prime 2} S_{12}^{(0)}\left(\mu^{\prime}, \mu_{0}\right)+S_{22}^{(0)}\left(\mu^{\prime}, \mu_{0}\right)\right]\right\} \\
& +2\left\{1-\mu^{2}+\frac{3}{8} \int_{0}^{1} \frac{d \mu^{\prime}}{\mu^{\prime}}\left(1-\mu^{\prime 2}\right) S_{11}^{(0)}\left(\mu, \mu^{\prime}\right)\right\} \\
& \times\left\{\frac{3}{8} \int_{0}^{1} \frac{d \mu^{\prime}}{\mu^{\prime}}\left(1-\mu^{\prime 2}\right) S_{12}^{(0)}\left(\mu^{\prime}, \mu_{0}\right)\right\} \text {; }
\end{aligned}
$$




$$
\begin{aligned}
\left(\frac{1}{\mu}+\right. & \left.\frac{1}{\mu_{0}}\right) S_{21}^{(0)}\left(\mu, \mu_{0}\right) \\
= & \left\{\mu_{0}^{2}+\frac{3}{8} \int_{0}^{1} \frac{d \mu^{\prime}}{\mu^{\prime}}\left[\mu^{\prime 2} S_{11}^{(0)}\left(\mu^{\prime}, \mu_{0}\right)+S_{21}^{(0)}\left(\mu^{\prime}, \mu_{0}\right)\right]\right\} \\
& \times\left\{1+\frac{3}{8} \int_{0}^{1} \frac{d \mu^{\prime}}{\mu^{\prime}}\left[\mu^{\prime 2} S_{21}^{(0)}\left(\mu, \mu^{\prime}\right)+S_{22}^{(0)}\left(\mu, \mu^{\prime}\right)\right]\right\} \\
& +2\left\{1-\mu_{0}^{2}+\frac{3}{8} \int_{0}^{1} \frac{d \mu^{\prime}}{\mu^{\prime}}\left(1-\mu^{\prime 2}\right) S_{11}^{(0)}\left(\mu^{\prime}, \mu_{0}\right)\right\} \\
& \times\left\{\frac{3}{8} \int_{0}^{1} \frac{d \mu^{\prime}}{\mu^{\prime}}\left(1-\mu^{\prime 2}\right) S_{21}^{(0)}\left(\mu, \mu^{\prime}\right)\right\} ;
\end{aligned}
$$

$$
\begin{aligned}
& \left(\frac{1}{\mu}+\frac{1}{\mu_{0}}\right) S_{22}^{(0)}\left(\mu, \mu_{0}\right) \\
& \quad=\left\{1+\frac{3}{8} \int_{0}^{1} \frac{d \mu^{\prime}}{\mu^{\prime}}\left[\mu^{\prime 2} S_{12}^{(0)}\left(\mu^{\prime}, \mu_{0}\right)+S_{22}^{(0)}\left(\mu^{\prime}, \mu_{0}\right)\right]\right\}
\end{aligned}
$$

$$
\begin{aligned}
& \times\left\{1+\frac{3}{8} \int_{0}^{1} \frac{d \mu^{\prime}}{\mu^{\prime}}\left[\mu^{\prime 2} S_{21}^{(0)}\left(\mu, \mu^{\prime}\right)+S_{22}^{(0)}\left(\mu, \mu^{\prime}\right)\right]\right\} \\
& +\frac{9}{32} \int_{0}^{1} \frac{d \mu^{\prime}}{\mu^{\prime}}\left(1-\mu^{\prime 2}\right) S_{21}^{(0)}\left(\mu, \mu^{\prime}\right) \\
& \times \int_{0}^{1} \frac{d \mu^{\prime}}{\mu^{\prime}}\left(1-\mu^{\prime 2}\right) S_{12}^{(0)}\left(\mu^{\prime}, \mu_{0}\right) .
\end{aligned}
$$

An inspection of these equations shows what we have already seen from the functional equation for $S$, that among these functions of zero-order the relation (182) must hold in particular. In other words

$$
\begin{gathered}
S_{11}^{(0)}\left(\mu, \mu_{0}\right)=S_{11}^{(0)}\left(\mu_{0}, \mu\right) ; \quad S_{12}^{(0)}\left(\mu, \mu_{0}\right)=S_{21}^{(0)}\left(\mu_{0}, \mu\right) ; \\
S_{22}^{(0)}\left(\mu, \mu_{0}\right)=S_{22}^{(0)}\left(\mu_{0}, \mu\right) .
\end{gathered}
$$

In view of these relations, it follows from equations (183)-(186) that we can express the functions $S_{11}^{(0)}, S_{12}^{(0)}, S_{21}^{(0)}$ and $S_{22}^{(0)}$ in the forms

$$
\begin{aligned}
& \left(\frac{1}{\mu}+\frac{1}{\mu_{0}}\right) S_{11}^{(0)}\left(\mu, \mu_{0}\right)=\psi(\mu) \psi\left(\mu_{0}\right)+2 \phi(\mu) \phi\left(\mu_{0}\right), \\
& \left(\frac{1}{\mu}+\frac{1}{\mu_{0}}\right) S_{12}^{(0)}\left(\mu, \mu_{0}\right)=\psi(\mu) \chi\left(\mu_{0}\right)+2 \phi(\mu) \zeta\left(\mu_{0}\right),
\end{aligned}
$$




$$
\left(\frac{1}{\mu}+\frac{1}{\mu_{0}}\right) S_{21}^{(0)}\left(\mu, \mu_{0}\right)=\psi\left(\mu_{0}\right) \chi(\mu)+2 \phi\left(\mu_{0}\right) \zeta(\mu),
$$

and

$$
\left(\frac{1}{\mu}+\frac{1}{\mu_{0}}\right) S_{22}^{(0)}\left(\mu, \mu_{0}\right)=\chi(\mu) \chi\left(\mu_{0}\right)+2 \zeta(\mu) \zeta\left(\mu_{0}\right),
$$

where

$$
\begin{aligned}
& \psi(\mu)=\mu^{2}+\frac{3}{8} \int_{0}^{1} \frac{d \mu^{\prime}}{\mu^{\prime}}\left[\mu^{\prime 2} S_{11}^{(0)}\left(\mu, \mu^{\prime}\right)+S_{12}^{(0)}\left(\mu, \mu^{\prime}\right)\right], \\
& \phi(\mu)=1-\mu^{2}+\frac{3}{8} \int_{0}^{1} \frac{d \mu^{\prime}}{\mu^{\prime}}\left(1-\mu^{\prime 2}\right) S_{11}^{(0)}\left(\mu, \mu^{\prime}\right), \\
& \chi(\mu)=1+\frac{3}{8} \int_{0}^{1} \frac{d \mu^{\prime}}{\mu^{\prime}}\left[\mu^{\prime 2} S_{21}^{(0)}\left(\mu, \mu^{\prime}\right)+S_{22}^{(0)}\left(\mu, \mu^{\prime}\right)\right]
\end{aligned}
$$

and

$$
\zeta(\mu)=\frac{3}{8} \int_{0}^{1} \frac{d \mu^{\prime}}{\mu^{\prime}}\left(1-\mu^{\prime 2}\right) S_{21}^{(0)}\left(\mu, \mu^{\prime}\right) .
$$

Substituting for $S_{11}^{(0)}$, and so on, from equations (188)-(191) back into equations (192)-(195) we obtain the functional equations for the problem in their normal forms. We have

$$
\begin{aligned}
& \psi(\mu)=\mu^{2}+\frac{3}{8} \mu \psi(\mu) \int_{0}^{1} \frac{d \mu^{\prime}}{\mu+\mu^{\prime}}\left[\mu^{\prime 2} \psi\left(\mu^{\prime}\right)+\chi\left(\mu^{\prime}\right)\right] \\
&+\frac{3}{4} \mu \phi(\mu) \int_{0}^{1} \frac{d \mu^{\prime}}{\mu+\mu^{\prime}}\left[\mu^{\prime 2} \phi\left(\mu^{\prime}\right)+\zeta\left(\mu^{\prime}\right)\right], \\
& \phi(\mu)=1-\mu^{2}+\frac{3}{8} \mu \psi(\mu) \int_{0}^{1} \frac{d \mu^{\prime}}{\mu+\mu^{\prime}}\left(1-\mu^{\prime 2}\right) \psi\left(\mu^{\prime}\right) \\
&+\frac{3}{4} \mu \phi(\mu) \int_{0}^{1} \frac{d \mu^{\prime}}{\mu+\mu^{\prime}}\left(1-\mu^{\prime 2}\right) \phi\left(\mu^{\prime}\right), \\
& \chi(\mu)=1+\frac{3}{8} \mu \chi(\mu) \int_{0}^{1} \frac{d \mu^{\prime}}{\mu+\mu^{\prime}}\left[\mu^{\prime 2} \psi\left(\mu^{\prime}\right)+\chi\left(\mu^{\prime}\right)\right] \\
&+\frac{3}{4} \mu \zeta(\mu) \int_{0}^{1} \frac{d \mu^{\prime}}{\mu+\mu^{\prime}}\left[\mu^{\prime 2} \phi\left(\mu^{\prime}\right)+\zeta\left(\mu^{\prime}\right)\right],
\end{aligned}
$$




$$
\begin{aligned}
\zeta(\mu)=\frac{3}{8} \mu \chi(\mu) & \int_{0}^{1} \frac{d \mu^{\prime}}{\mu+\mu^{\prime}}\left(1-\mu^{\prime 2}\right) \psi\left(\mu^{\prime}\right) \\
& +\frac{3}{4} \mu \zeta(\mu) \int_{0}^{1} \frac{d \mu^{\prime}}{\mu+\mu^{\prime}}\left(1-\mu^{\prime 2}\right) \phi\left(\mu^{\prime}\right) .
\end{aligned}
$$

31. The solution of the functional equations (196)-(199). Equations (196)-(199) represent a nonlinear, nonhomogeneous system of four simultaneous functional equations which one might despair of even attempting to solve. However, with the guidance provided by the form of the solution obtained in our general $n$th approximation, it is possible to reduce the solution of equations (196)-(199) to two simple functional equations, each of form (168). Thus, it can be verified by direct substitution that the solution of the system of equations (196)-(199) is given by

$$
\begin{aligned}
& \psi(\mu)=\left(2\left(1-c^{2}\right)\right)^{1 / 2} \mu H_{l}(\mu), \\
& \phi(\mu)=H_{l}(\mu)(1-c \mu), \\
& \chi(\mu)=H_{r}(\mu)(1+c \mu),
\end{aligned}
$$

and

$$
\zeta(\mu)=\frac{\left(1-c^{2}\right)^{1 / 2}}{2^{1 / 2}} \mu H_{r}(\mu),
$$

where $H_{l}(\mu)$ and $H_{r}(\mu)$ are defined in terms of the functional equations

$$
H_{l}(\mu)=1+\frac{3}{4} \mu H_{l}(\mu) \int_{0}^{1} \frac{H_{l}\left(\mu^{\prime}\right)}{\mu+\mu^{\prime}}\left(1-\mu^{\prime 2}\right) d \mu^{\prime}
$$

and

$$
H_{r}(\mu)=1+\frac{3}{8} \mu H_{r}(\mu) \int_{0}^{1} \frac{H_{r}\left(\mu^{\prime}\right)}{\mu+\mu^{\prime}}\left(1-\mu^{\prime 2}\right) d \mu^{\prime},
$$

and $c$ is a constant related in a determinate way with the moments of $H_{l}(\mu)$ and $H_{r}(\mu)$. We find

$$
c=\frac{8\left(A_{1}-\alpha_{1}\right)+3\left(2 \alpha_{1} \alpha_{0}-A_{1} A_{0}\right)}{3\left(A_{1}^{2}+2 \alpha_{1}^{2}\right)},
$$

where $\alpha_{0}, A_{0}$ and $\alpha_{1}, A_{1}$ are the moments of order zero and one of $H_{l}(\mu)$ and $H_{r}(\mu)$, respectively.

With $\psi(\mu), \phi(\mu)$, and so on, defined as in equations (200)-(203) we can verify that the solutions for $S_{11}^{(0)}$, and so on, are in entire agree- 
ment with our earlier results obtained by passing to the limit of infinite approximation.

32. The completion of the solution for $\boldsymbol{S}$. The discussion of the other two systems for the functions of order one and two turns out to be very simple, as it appears that all the functions $S^{(1)}$ are equal to each other and similarly all the functions $S^{(2)}$ are equal to each other. Therefore, writing

$$
S_{i, j}^{(1)}\left(\mu, \mu_{0}\right)=S^{(1)}\left(\mu, \mu_{0}\right),
$$

and

$$
S_{i, j}^{(2)}\left(\mu, \mu_{0}\right)=S^{(2)}\left(\mu, \mu_{0}\right),
$$

it is found that the equations governing $S^{(1)}$ and $S^{(2)}$ are

$$
\begin{aligned}
\left(\frac{1}{\mu}+\frac{1}{\mu_{0}}\right) & S^{(1)}\left(\mu, \mu_{0}\right) \\
= & \left\{1+\frac{3}{8} \int_{0}^{1} \frac{d \mu^{\prime}}{\mu^{\prime}}\left(1-\mu^{\prime 2}\right)\left(1+2 \mu^{\prime 2}\right) S^{(1)}\left(\mu^{\prime}, \mu_{0}\right)\right\} \\
& \times\left\{1+\frac{3}{8} \int_{0}^{1} \frac{d \mu^{\prime}}{\mu^{\prime}}\left(1-\mu^{\prime 2}\right)\left(1+2 \mu^{\prime 2}\right) S^{(1)}\left(\mu, \mu^{\prime}\right)\right\},
\end{aligned}
$$

and

$$
\left(\frac{1}{\mu}+\frac{1}{\mu_{0}}\right) S^{(2)}\left(\mu, \mu_{0}\right)=\left\{1+\frac{3}{16} \int_{0}^{1} \frac{d \mu^{\prime}}{\mu^{\prime}}\left(1+\mu^{\prime 2}\right)^{2} S^{(2)}\left(\mu^{\prime}, \mu_{0}\right)\right\}
$$

$$
\times\left\{1+\frac{3}{16} \int_{0}^{1} \frac{d \mu^{\prime}}{\mu^{\prime}}\left(1+\mu^{\prime 2}\right)^{2} S^{(2)}\left(\mu, \mu^{\prime}\right)\right\} .
$$

From these equations it follows that the functions $S^{(1)}\left(\mu, \mu_{0}\right)$ and $S^{(2)}\left(\mu, \mu_{0}\right)$ are symmetrical in the variables $\mu$ and $\mu_{0}$ and that they are expressible in the forms

$$
\left(\frac{1}{\mu}+\frac{1}{\mu_{0}}\right) S^{(1)}\left(\mu, \mu_{0}\right)=H^{(1)}(\mu) H^{(1)}\left(\mu_{0}\right),
$$

and

$$
\left(\frac{1}{\mu}+\frac{1}{\mu_{0}}\right) S^{(2)}\left(\mu, \mu_{0}\right)=H^{(2)}(\mu) H^{(2)}\left(\mu_{0}\right)
$$


where $H^{(1)}(\mu)$ and $H^{(2)}(\mu)$ are solutions of the functional equations

$$
H^{(1)}(\mu)=1+\frac{3}{8} \mu H^{(1)}(\mu) \int_{0}^{1} \frac{H^{(1)}\left(\mu^{\prime}\right)}{\mu+\mu^{\prime}}\left(1-\mu^{\prime 2}\right)\left(1+2 \mu^{\prime 2}\right) d \mu^{\prime},
$$

and

$$
H^{(2)}(\mu)=1+\frac{3}{16} \mu H^{(2)}(\mu) \int_{0}^{1} \frac{H^{(2)}\left(\mu^{\prime}\right)}{\mu+\mu^{\prime}}\left(1+\mu^{\prime 2}\right)^{2} d \mu^{\prime} .
$$

With this, the solution of the functional equation for $S$ is completed and it will be observed that the solution for $S$ which we have now obtained is of exactly the same form as that given in $\$ 22$ (under D) with the only difference that the $H$-functions which appear in the solution are now defined in terms of the exact functional equations which they satisfy; further, the constant $c$ is shown to be related in a definite way with the moments of $H_{l}(\mu)$ and $H_{r}(\mu)$.

33. A class of functional equations and their solution. The solution of the functional equations for the problem of diffuse reflection for laws of scattering, other than the one we have described, can be carried out in an analogous manner. It is not our intention to go into the details of the solution of these other cases here, but it may be of some mathematical interest to see the type of functional equations which these problems lead us to consider.

The problem of diffuse reflection in accordance with Rayleigh's phase function leads to the following simultaneous pair of functional equations:

$$
\begin{aligned}
\psi(\mu)=3-\mu^{2} & +\frac{1}{16} \mu \psi(\mu) \int_{0}^{1} \frac{\psi\left(\mu^{\prime}\right)}{\mu+\mu^{\prime}}\left(3-\mu^{\prime 2}\right) d \mu^{\prime} \\
& +\frac{1}{2} \mu \phi(\mu) \int_{0}^{1} \frac{\phi\left(\mu^{\prime}\right)}{\mu+\mu^{\prime}}\left(3-\mu^{\prime 2}\right) d \mu^{\prime}
\end{aligned}
$$

and

$$
\begin{aligned}
\phi(\mu)=\mu^{2} & +\frac{1}{16} \mu \psi(\mu) \int_{0}^{1} \frac{\psi\left(\mu^{\prime}\right)}{\mu+\mu^{\prime}} \mu^{\prime 2} d \mu^{\prime} \\
& +\frac{1}{2} \mu \phi(\mu) \int_{0}^{1} \frac{\phi\left(\mu^{\prime}\right)}{\mu+\mu^{\prime}} \mu^{\prime 2} d \mu^{\prime}
\end{aligned}
$$

Again, guided by the form of the solution obtained by the direct solution of the equation of transfer (see the tabulation in \$22, under B), we are led to surmise that the solutions of equations (215) and 
(216) must be of the forms

$$
\psi(\mu)=H^{(0)}(\mu)(3-c \mu)
$$

and

$$
\phi(\mu)=q \mu H^{(0)}(\mu),
$$

where $H^{(0)}(\mu)$ satisfies the functional equation

$$
H^{(0)}(\mu)=1+\frac{3}{16} \mu H^{(0)}(\mu) \int_{0}^{1} \frac{H^{(0)}\left(\mu^{\prime}\right)}{\mu+\mu^{\prime}}\left(3-\mu^{\prime 2}\right) d \mu^{\prime},
$$

and $q$ and $c$ are two constants related in the manner

$$
8 q^{2}=3-c^{2}
$$

Direct substitution confirms that the solutions of equations (215) and (216) are indeed of the forms surmised and shows further that, in agreement with equation (165),

$$
q=\frac{2}{3 \alpha_{1}}
$$

where $\alpha_{1}$ is the first moment of $H^{(0)}(\mu)$.

Similarly, the problem of diffuse reflection in accordance with the phase function $\lambda(1+x \cos \Theta)$ leads to the following pair of functional equations:

$$
\begin{aligned}
\psi(\mu)=1 & +\frac{1}{2} \lambda \mu \psi(\mu) \int_{0}^{1} \frac{\psi\left(\mu^{\prime}\right)}{\mu+\mu^{\prime}} d \mu^{\prime} \\
& -\frac{1}{2} x \lambda \mu \phi(\mu) \int_{0}^{1} \frac{\phi\left(\mu^{\prime}\right)}{\mu+\mu^{\prime}} d \mu^{\prime},
\end{aligned}
$$

and

$$
\begin{aligned}
\phi(\mu)=\mu & -\frac{1}{2} \lambda \mu \psi(\mu) \int_{0}^{1} \frac{\psi\left(\mu^{\prime}\right)}{\mu+\mu^{\prime}} \mu^{\prime} d \mu^{\prime} \\
& +\frac{1}{2} x \lambda \mu \phi(\mu) \int_{0}^{1} \frac{\phi\left(\mu^{\prime}\right)}{\mu+\mu^{\prime}} \mu^{\prime} d \mu^{\prime} .
\end{aligned}
$$

And, it is found by direct verification that the solution of these equations can be expressed in the form

$$
\psi(\mu)=H^{(0)}(\mu)(1-c \mu),
$$

and 


$$
\phi(\mu)=q \mu H^{(0)}(\mu),
$$

where $H^{(0)}(\mu)$ now satisfies the functional equation

$$
H^{(0)}(\mu)=1+\frac{1}{2} \lambda \mu H^{(0)}(\mu) \int_{0}^{1} \frac{H^{(0)}\left(\mu^{\prime}\right)}{\mu+\mu^{\prime}}\left[1+x(1-\lambda) \mu^{\prime 2}\right] d \mu^{\prime},
$$

and $q$ and $c$ are two constants related in the manner

$$
x q^{2}=c^{2}+x(1-\lambda),
$$

and given explicitly by the formulae

$$
q=\frac{2(1-\lambda)}{2-\lambda \alpha_{0}} \text { and } c=x \lambda(1-\lambda) \frac{\alpha_{1}}{2-\lambda \alpha_{0}},
$$

$\alpha_{0}$ and $\alpha_{1}$ being the moments of order zero and one of $H^{(0)}(\mu)$.

34. Some general remarks. In some ways it is remarkable that systems of functional equations as complex in appearance as equations (196)-(199), (215)-(216) and (222)-(223) are should be capable of being reduced to single functional equations of the form (168). There must clearly be something in the structure of these equations which makes this reduction possible. But, as to what it precisely is, is at present shrouded in mystery!

35. Some integral properties of the functions $H(\mu)$. The discussion in the preceding sections has disclosed the important role which functional equations of the form

$$
H(\mu)=1+\mu H(\mu) \int_{0}^{1} \frac{H\left(\mu^{\prime}\right)}{\mu+\mu^{\prime}} \Psi\left(\mu^{\prime}\right) d \mu^{\prime}
$$

play in the theory of radiative transfer. The investigation of the properties of these equations is, therefore, a matter of considerable interest. Of course, from the practical standpoint of solving such equations numerically, the most important property is that derived from Theorem 1 ( $\$ 24)$, namely, that when we replace the integral on the righthand side by a Gauss sum, the solution can be explicitly written down as a rational function involving the points of the Gaussian division and the roots of the associated characteristic equation

$$
1=2 \sum_{j=1}^{n} \frac{a_{j} \Psi\left(\mu_{j}\right)}{1-k^{2} \mu_{j}^{2}} .
$$

However, in addition to this property, there are a number of integral theorems (of an essentially elementary kind) which can be proved for 
functions satisfying equations of the form (229). We shall give two examples.

Theorem 3. $\int_{0}^{1} H(\mu) \Psi(\mu) d \mu=1-\left[1-2 \int_{0}^{1} \Psi(\mu) d \mu\right]^{1 / 2}$.

Proof. Multiplying the equation satisfied by $H(\mu)$ by $\Psi(\mu)$ and integrating over the range of $\mu$, we have

$$
\begin{aligned}
& \int_{0}^{1} H(\mu) \Psi(\mu) d \mu \\
& \quad=\int_{0}^{1} \Psi(\mu) d \mu+\int_{0}^{1} \int_{0}^{1} \frac{\mu}{\mu+\mu^{\prime}} H(\mu) \Psi(\mu) H\left(\mu^{\prime}\right) \Psi\left(\mu^{\prime}\right) d \mu d \mu^{\prime} .
\end{aligned}
$$

Interchanging $\mu$ and $\mu^{\prime}$ in the double integral in equation (231) and taking the average of the two equations, we obtain

$$
\begin{aligned}
& \int_{0}^{1} H(\mu) \Psi(\mu) d \mu \\
& \quad=\int_{0}^{1} \Psi(\mu) d \mu+\frac{1}{2} \int_{0}^{1} \int_{0}^{1} H(\mu) \Psi(\mu) H\left(\mu^{\prime}\right) \Psi\left(\mu^{\prime}\right) d \mu d \mu^{\prime}
\end{aligned}
$$

or, alternatively,

$$
\frac{1}{2}\left[\int_{0}^{1} H(\mu) \Psi(\mu) d \mu\right]^{2}-\int_{0}^{1} H(\mu) \Psi(\mu) d \mu+\int_{0}^{1} \Psi(\mu) d \mu=0 .
$$

Solving this equation for the integral in question, we have

$$
\int_{0}^{1} H(\mu) \psi(\mu) d \mu=1 \pm\left[1-2 \int_{0}^{1} \Psi(\mu) d \mu\right]^{1 / 2} .
$$

The ambiguity in the sign in equation (234) can be removed by the consideration that the integral on the left-hand side must uniformly converge to zero when $\Psi(\mu)$ tends to zero uniformly in the interval $(0,1)$. This requires us to choose the negative sign in equation (234) and the result stated follows.

Corollary. A necessary and sufficient condition that $H(\mu)$ be real is

$$
\int_{0}^{1} \Psi(\mu) d \mu \leqq \frac{1}{2} .
$$

This is, of course, an immediate consequence of the theorem.

The physical meaning of the limitation on $\Psi(\mu)$ implied by this corollary is interesting: it is really equivalent to the condition that, 
on each scattering, more radiation should not be emitted than was incident; further, the equality sign is admissible only in the case of perfect scattering in the sense of equation (11).

THEOREM 4. $\left[1-2 \int_{0}^{1} \Psi(\mu) d \mu\right]^{1 / 2} \int_{0}^{1} H(\mu) \Psi(\mu) \mu^{2} d \mu+\left[\int_{0}^{1} H(\mu) \Psi(\mu) \mu d \mu\right]^{2} / 2$ $=\int_{0}^{1} \Psi(\mu) \mu^{2} d \mu$.

Proof. To prove this theorem, we multiply the equation defining $H(\mu)$ by $\Psi(\mu) \mu^{2}$ and integrate over the range of $\mu$. We find

$$
\begin{array}{rl}
\int_{0}^{1} & H(\mu) \Psi(\mu) \mu^{2} d \mu \\
= & \int_{0}^{1} \Psi(\mu) \mu^{2} d \mu+\int_{0}^{1} \int_{0}^{1} \frac{H(\mu) H\left(\mu^{\prime}\right) \Psi(\mu) \Psi\left(\mu^{\prime}\right)}{\mu+\mu^{\prime}} \mu^{3} d \mu d \mu^{\prime} \\
= & \int_{0}^{1} \Psi(\mu) \mu^{2} d \mu \\
& +\frac{1}{2} \int_{0}^{1} \int_{0}^{1} \frac{H(\mu) H\left(\mu^{\prime}\right) \Psi(\mu) \Psi\left(\mu^{\prime}\right)}{\mu+\mu^{\prime}}\left(\mu^{3}+\mu^{\prime 3}\right) d \mu d \mu^{\prime} \\
= & \int_{0}^{1} \Psi(\mu) \mu^{2} d \mu \\
& +\frac{1}{2} \int_{0}^{1} \int_{0}^{1} H(\mu) H\left(\mu^{\prime}\right) \Psi(\mu) \Psi\left(\mu^{\prime}\right)\left(\mu^{2}-\mu \mu^{\prime}+\mu^{\prime 2}\right) d \mu d \mu^{\prime} \\
= & \int_{0}^{1} \Psi(\mu) \mu^{2} d \mu+\left[\int_{0}^{1} H(\mu) \Psi(\mu) \mu^{2} d \mu\right]\left[\int_{0}^{1} H(\mu) \Psi(\mu) d \mu\right] \\
& -\frac{1}{2}\left[\int_{0}^{1} H(\mu) \Psi(\mu) \mu d \mu\right]^{2} .
\end{array}
$$

Using Theorem 3 we obtain, after some minor reductions,

$$
\begin{aligned}
{\left[1-2 \int_{0}^{1} \Psi(\mu) d \mu\right]^{1 / 2} } & \int_{0}^{1} H(\mu) \Psi(\mu) \mu^{2} d \mu \\
+ & \frac{1}{2}\left[\int_{0}^{1} H(\mu) \Psi(\mu) \mu d \mu\right]^{2}=\int_{0}^{1} \Psi(\mu) \mu^{2} d \mu,
\end{aligned}
$$

which is the required result.

COROLLARY. For the case of a perfectly scattering atmosphere when

$$
\int_{0}^{1} \Psi(\mu) d \mu=1 / 2,
$$


we have the further integral

$$
\int_{0}^{1} H(\mu) \Psi(\mu) \mu d \mu=\left[2 \int_{0}^{1} \Psi(\mu) \mu^{2} d \mu\right]^{1 / 2} .
$$

The corollary we have just stated generalizes a classical result of Hopf and Bronstein for the case of an isotropically scattering atmosphere. For, in this latter case

$$
\Psi(\mu)=\text { constant }=1 / 2,
$$

and, according to Theorem 3 and the corollary of Theorem 4 , we have

$$
\begin{gathered}
\int_{0}^{1} H(\mu) d \mu=2, \\
\int_{0}^{1} H(\mu) \mu d \mu=\frac{2}{3^{1 / 2}} .
\end{gathered}
$$

Hence

$$
\frac{J(0)}{F}=\frac{\int_{0}^{1} H(\mu) d \mu}{4 \int_{0}^{1} H(\mu) \mu d \mu}=\frac{3^{1 / 2}}{4} .
$$

This is the Hopf-Bronstein relation. It, therefore, follows that for all cases of perfect scattering we have an integral of the Hopf-Bronstein type which is essentially that given by the corollary of Theorem 4.

36. The equation of transfer in spherical atmospheres, and its reduction for the case $\kappa \rho \alpha r^{-n}$. So far we have restricted ourselves to transfer problems in plane parallel atmospheres. We shall now briefly indicate how the methods we have described can be extended to treat transfer problems in spherically symmetric atmospheres. In such cases, the intensity in the radiation field will be a function of the distance $r$ from the center of symmetry and the angle $\vartheta$ measured from the positive direction of the radius vector; and the equation of transfer will take the form

$$
\mu \frac{\partial I}{\partial r}+\frac{1-\mu^{2}}{r} \frac{\partial I}{\partial \mu}=-\kappa \rho(I-\Im),
$$

where $\mu=\cos \vartheta$ and $\Im$ denotes, as usual, the source function.

In outlining the manner in which equations of transfer of the form (241) can be solved, we shall restrict ourselves to an isotropically 
scattering atmosphere. In this case equation (241) becomes

$$
\mu \frac{\partial I}{\partial r}+\frac{1-\mu^{2}}{r} \frac{\partial I}{\partial \mu}=-\kappa \rho I+\frac{1}{2} \kappa \rho \int_{-1}^{+1} I\left(r, \mu^{\prime}\right) d \mu^{\prime} .
$$

According to the ideas developed in the earlier parts of this lecture, we shall replace the integral which occurs on the right-hand side of equation (242) by a sum according to Gauss's formula for numerical quadratures, and reduce the integrodifferential equation to the system

$$
\begin{aligned}
\mu_{i} \frac{d I_{i}}{d r}+\frac{1-\mu_{i}^{2}}{r}\left(\frac{\partial I}{\partial \mu}\right)_{\mu=\mu_{i}}=-\kappa \rho I_{i}+ & \frac{1}{2} \kappa \rho \sum a_{j} I_{i} \\
& (i= \pm 1, \cdots, \pm n)
\end{aligned}
$$

of $2 n$ ordinary linear equations in the $n$th approximation. It is at once seen that our present system of equations differs, in an essential way, from those which we have considered so far: equation (243) now involves $(\partial I / \partial \mu)_{\mu=\mu_{i}}$ and, before we can proceed any further, we must know the values which we are to assign to $\partial I / \partial \mu$ at the points of the Gaussian division in our present scheme of approximation. At first sight it might be supposed that the assignment of values to $\partial I / \partial \mu$ at $\mu=\mu_{i}, i= \pm 1, \cdots, \pm n$, is largely arbitrary, particularly when $n$ is small. However, on consideration, it appears that this assignment can be done in a satisfactory manner in only one way and, indeed, according to the following device:

Define the polynomials $Q_{m}(\mu)$ according to the formula

$$
P_{m}(\mu)=-\frac{d Q_{m}}{d \mu} \quad(m=1, \cdots, 2 n),
$$

and adjust the constant of integration in $Q_{m}$ by requiring that

$$
Q_{m}=0
$$$$
\text { for }|\mu|=1 \text {. }
$$

This can always be accomplished, since, when $m$ is odd, $Q_{m}$ is even and when $m$ is even the indefinite integral of $P_{m}(\mu)$ already contains $\left(1-\mu^{2}\right)$ as a factor. The first few polynomials $Q_{m}(\mu)$ are given below:

\begin{tabular}{llll}
$m$ & \multicolumn{1}{c}{$P_{m}(\mu)$} & \multicolumn{1}{c}{$Q_{m}(\mu)$} & \multicolumn{1}{c}{$\mathfrak{Q}_{m}(\mu)$} \\
1 & $\mu$ & $\left(1-\mu^{2}\right) / 2$ & $1 / 2$ \\
2 & $\left(3 \mu^{2}-1\right) / 2$ & $\mu\left(1-\mu^{2}\right) / 2$ & $\mu / 2$ \\
3 & $\left(5 \mu^{3}-3 \mu\right) / 2$ & $1 / 8\left(5 \mu^{2}-1\right)\left(1-\mu^{2}\right)$ & $1 / 8\left(5 \mu^{2}-1\right)$ \\
4 & $1 / 8\left(35 \mu^{4}-30 \mu^{2}+3\right)$ & $1 / 8 \mu\left(7 \mu^{2}-3\right)\left(1-\mu^{2}\right)$ & $1 / 8 \mu\left(7 \mu^{2}-3\right)$ \\
5 & $1 / 8\left(63 \mu^{5}-70 \mu^{3}+15 \mu\right)$ & $1 / 16\left(21 \mu^{4}-14 \mu^{2}+1\right)\left(1-\mu^{2}\right)$ & $1 / 16\left(21 \mu^{4}-14 \mu^{2}+1\right)$
\end{tabular}


Now, by an integration by parts, we arrive at the identity

$$
\int_{-1}^{+1} Q_{m}(\mu) \frac{\partial I}{\partial \mu} d \mu=-\int_{-1}^{+1} I \frac{d Q_{m}}{d \mu} d \mu=\int_{-1}^{+1} I P_{m}(\mu) d \mu .
$$

Expressing the first and the last integrals in equation (246) as sums according to Gauss's formula, we have, in the $n$th approximation,

$$
\sum a_{i} Q_{m}\left(\mu_{i}\right)\left(\frac{\partial I}{\partial \mu}\right)_{\mu=\mu_{i}}=\sum a_{i} I_{i} P_{m}\left(\mu_{i}\right) \quad(m=1, \cdots, 2 n) .
$$

Equation (247) provides us with exactly the right number of equations to express $(\partial I / \partial \mu)_{\mu=\mu_{i}}(i= \pm 1, \cdots, \pm n)$ as linear combinations of $I_{i}$. Essentially what equation (247) allows is to determine in a "best possible way" the derivatives of a function in terms of its values at the points of the Gaussian division. This problem has apparently not been considered before.

Returning to equation (243) we now observe that this equation, together with equation (246), provides the required reduction of the equation of transfer (242) to an equivalent system of linear equations.

For purposes of practical solution it appears most convenient to combine equations (243) and (247) in the following manner.

Since we have arranged $Q_{m}(\mu)$ to be divisible by $\left(1-\mu^{2}\right)$, we can clearly write

$$
Q_{m}(\mu)=\mathfrak{Q}_{m}(\mu)\left(1-\mu^{2}\right) .
$$

The first few of the polynomials $\mathfrak{Q}_{m}(\mu)$ are listed in the preceding tabulation.

Now, multiply equation (243) by $a_{i} \mathfrak{Q}_{m}\left(\mu_{i}\right)$ and sum over all $i$ 's. We obtain

$$
\begin{array}{r}
\frac{d}{d r} \sum a_{i} \mu_{i} \mathfrak{Q}_{m}\left(\mu_{i}\right) I_{i}+\frac{1}{r} \sum a_{i}\left(1-\mu_{i}^{2}\right) \mathfrak{Q}_{m}\left(\mu_{i}\right)\left(\frac{\partial I}{\partial \mu}\right)_{\mu=\mu_{i}} \\
=-\kappa \rho \sum a_{i} \bigcap_{m}\left(\mu_{i}\right) I_{i}+\frac{1}{2} \kappa \rho\left(\sum a_{i} I_{i}\right)\left[\sum a_{i} \mathfrak{Q}_{m}\left(\mu_{i}\right)\right] \\
(m=1, \cdots, 2 n) .
\end{array}
$$

But, according to equations (247) and (248)

$$
\begin{aligned}
\sum a_{i}\left(1-\mu_{i}^{2}\right) \Omega_{m}\left(\mu_{i}\right)\left(\frac{\partial I}{\partial \mu}\right)_{\mu=\mu_{i}} & =\sum a_{i} Q_{m}\left(\mu_{i}\right)\left(\frac{\partial I}{\partial \mu}\right)_{\mu=\mu_{i}} \\
& =\sum a_{i} P_{m}\left(\mu_{i}\right) I_{i} .
\end{aligned}
$$


Equation (249), therefore, reduces to

$$
\begin{aligned}
& \frac{d}{d r} \sum a_{i} \mu_{i} \mathfrak{Q}_{m}\left(\mu_{i}\right) I_{i}+\frac{1}{r} \sum a_{i} P_{m}\left(\mu_{i}\right) I_{i} \\
& =-\kappa \rho \sum a_{i} \mathfrak{Q}_{m}\left(\mu_{i}\right) I_{i}+\frac{1}{2} \kappa \rho\left(\sum a_{i} I_{i}\right)\left[\sum a_{i} \mathfrak{Q}_{m}\left(\mu_{i}\right)\right] \\
& \quad(m=1, \cdots, 2 n) .
\end{aligned}
$$

This is the required system of linear equations in the $n$th approximation.

Equation (251) for the case $m=1$ admits of immediate integration. For, when $m=1$

$$
P_{1}(\mu)=\mu \text { and } \mathfrak{Q}_{1}(\mu)=1 / 2,
$$

and equation (251) yields

$$
\frac{1}{2} \frac{d}{d r} \sum a_{i} \mu_{i} I_{i}+\frac{1}{r} \sum a_{i} \mu_{i} I_{i}=0
$$

or

$$
\sum a_{i} \mu_{i} I_{i}=\frac{1}{2} \frac{F_{0}^{1}}{r^{2}}
$$

where $F_{0}$ is a constant. This is the equivalent, in our approximation, of the flux integral

$$
F=2 \int_{-1}^{+1} I \mu d \mu=\frac{F_{0}}{r^{2}}
$$

which the equation of transfer (242) admits directly.

Again, since $\mathfrak{Q}_{m}(\mu)$ is odd when $m$ is even, equation (251) reduces for even values of $m$ to the form

$$
\begin{array}{r}
\frac{d}{d r} \sum a_{i} \mu_{i} \mathfrak{Q}_{m}\left(\mu_{i}\right) I_{i}+\frac{1}{r} \sum a_{i} P_{m}\left(\mu_{i}\right) I_{i}=-\kappa \rho \sum a_{i} \mathfrak{Q}_{m}\left(\mu_{i}\right) I_{i} \\
(m=2,4, \cdots, 2 n) .
\end{array}
$$

For $m=2 n$, the foregoing equation further simplifies to

$$
\frac{d}{d r} \sum a_{i} \mu_{i} \mathfrak{Q}_{2 n}\left(\mu_{i}\right) I_{i}=-\kappa \rho \sum a_{i} \mathfrak{Q}_{2 n}\left(\mu_{i}\right) I_{i}
$$

Finally, we may note the explicit forms of the equations in the sec- 
ond approximation. They are

$$
\begin{gathered}
\sum a_{i} \mu_{i} I_{i}=\frac{1}{2} \frac{F_{0}}{r^{2}} \\
\frac{d}{d r} \sum a_{i} \mu_{i}^{2} I_{i}+\frac{1}{r} \sum a_{i}\left(3 \mu_{i}^{2}-1\right) I_{i}=-\kappa \rho \sum a_{i} \mu_{i} I_{i}, \\
\text { (258) } \frac{d}{d r} \sum a_{i} \mu_{i}\left(5 \mu_{i}^{2}-1\right) I_{i}+\frac{4}{r} \sum a_{i} \mu_{i}\left(5 \mu_{i}^{2}-3\right) I_{i} \\
=-\frac{5}{3} \kappa \rho \sum a_{i}\left(3 \mu_{i}^{2}-1\right) I_{i}, \\
\frac{d}{d r} \sum a_{i} \mu_{i}^{2}\left(7 \mu_{i}^{2}-3\right) I_{i}=-\kappa \rho \sum a_{i} \mu_{i}\left(7 \mu_{i}^{2}-3\right) I_{i} .
\end{gathered}
$$

From the point of view of astrophysical applications, greatest interest is attached to the case when $\kappa \rho$ varies as some inverse of power. And when

$$
\kappa \rho=\frac{\text { constant }}{r^{n}} \quad(n>1),
$$

the equations of the second approximation (258) can be solved explicitly and the various physical quantities expressed as integrals over the Bessel functions, $I_{\nu}$ and $K_{\nu}$, of purely imaginary argument, and of order

$$
\nu=\frac{n+5}{2(n-1)} .
$$

It does not, however, seem that the passage to the limit of infinite approximation can be achieved as simply as in the case of transfer problems in plane parallel atmospheres.

37. The equation of transfer in a differentially moving atmosphere. The influence of Doppler effect. Finally, we shall turn to a class of transfer problems which is of an altogether different character from the ones we have considered so far. The problems in question arise in connection with the study of the transfer of radiation in atmospheres in which the different parts are in relative motion. To be specific, consider a plane-parallel atmosphere in which the material at height $z$ has a velocity $w\left(z_{1}\right)$ with respect to a stationary observer. The novelty of the situation arises on account of Doppler effect which makes the radiation scattered in different directions have different frequencies 
as judged by a stationary observer. Consequently, the radiation field in the different frequencies will interact with each other in a manner which is not always easy to visualize. However, in the astrophysical contexts, two circumstances simplify the problem. First, the velocities which are involved are small compared to the velocity of light, $c$, and second, the only effects of consequence are those which arise from the sensitive dependence of the scattering coefficient $\sigma(\nu)$ on frequency. This last circumstance, in particular, allows us to ignore all effects such as aberration and so on, and concentrate only on the effects arising from the change of frequency on scattering. Under these conditions, the equation of transfer can be shown to take the form

$$
\begin{aligned}
\mu \frac{\partial I(\nu, z, \mu)}{\rho \partial z}= & -\sigma\left(\nu-\nu_{0} \frac{w}{c} \mu\right)\{I(\nu, z, \mu) \\
& \left.-\frac{1}{2} \int_{-1}^{+1} I\left(\nu-\nu_{0} \frac{w}{c} \mu+\nu_{0} \frac{w}{c} \mu^{\prime}, z, \mu^{\prime}\right) d \mu^{\prime}\right\},
\end{aligned}
$$

where $\nu_{0}$ denotes the frequency of the "center of the line."

With suitable simplifying assumptions concerning $\sigma(\nu)$ and $w(z)$, the discussion of the equation of transfer (261), in the first approximation in our scheme of replacing integrals by Gauss sums, leads to a variety of novel types of boundary value problems in hyperbolic equations. It may be of some interest to specify the nature of these boundary value problems and indicate the methods which have been developed for their solution.

38. A new class of boundary value problems in hyperbolic equations. As related to the equation

$$
\frac{\partial^{2} f}{\partial x^{2}}-\frac{\partial^{2} f}{\partial y^{2}}+f=0
$$

the boundary value problems which are of most frequent occurrence are of the following general type:

The value of $f$ and its derivatives are assigned for

$$
y=0 \text { and } 0 \leqq x \leqq l_{1},
$$

that is, along $A B$ in Fig. 2. Along $A D\left(x=0\right.$ and $\left.0 \leqq y \leqq l_{2}\right)$ and $B C$ $\left(x=l_{1}, 0 \leqq y \leqq l_{2}\right)$, we are further given that

$$
\begin{array}{cc}
\left(\frac{\partial f}{\partial x}\right)_{x=0}=\left(\frac{\partial f}{\partial y}\right)_{x=0}+\phi(y) & \left(0 \leqq y \leqq l_{2}\right), \\
f\left(l_{1}, y\right)=\psi(y) & \left(0 \leqq y \leqq l_{2}\right)
\end{array}
$$


where $\phi(y)$ and $\psi(y)$ are two known functions. The problem is to solve equation (1) in the rectangular strip $A B C D$ satisfying the stated boundary conditions. For the particular boundary value problems which occur in the astrophysical contexts, the following "systematic method" of solution has been found convenient.

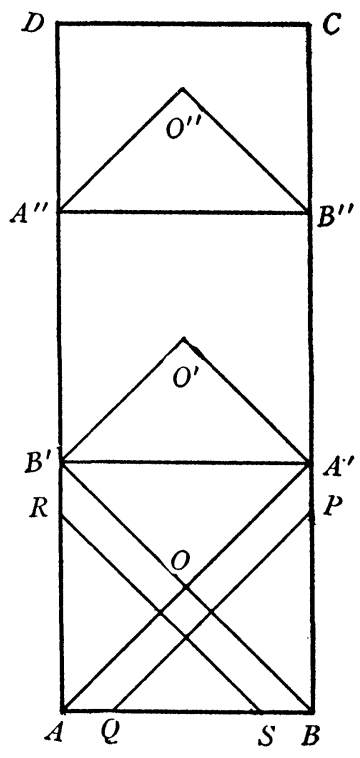

FIG. 2.

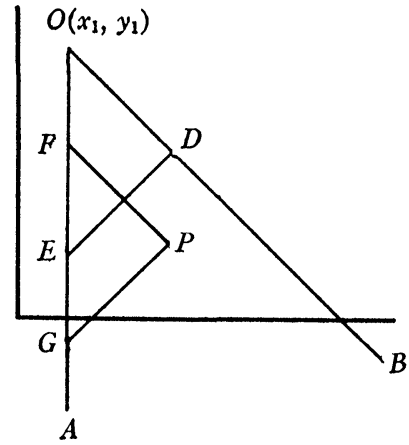

FIG. 3.

Let the characteristics $x=y$ and $l_{1}-x=y$ through $A$ and $B$ intersect $B C$ and $A D$ at $A^{\prime}$ and $B^{\prime}$, respectively. Further, let $A A^{\prime}$ and $B B^{\prime}$ intersect at $O$. First, since the function and its derivatives are known along $A B$, we can find the solution inside and on the sides of the triangle $O A B$ directly by Riemann's well known method. Next we use the requirement that the solution be unambiguously defined along $A O$ and $O B$ together with the boundary conditions specified along $A D$ and $B C$ to determine $f$ along $A B^{\prime}$ and $\partial f / \partial x$ along $B A^{\prime}$ as solutions of certain integral equations of Volterra's type. With the knowledge of the function and its derivatives thus completed along the part $B^{\prime} A B A^{\prime}$ of the "supporting curve" $A B C D$, the solution inside the entire region $O^{\prime} B^{\prime} A B A^{\prime}$ becomes determinate by Riemann's method. In particular, the function and its derivatives along $B^{\prime} A^{\prime}$ can be found and the continuation of the solution in the second square $B^{\prime} A^{\prime} B^{\prime \prime} A^{\prime \prime}$ follows along similar lines. 
While the method outlined above shows how solutions satisfying the given boundary conditions can be found in principle, it suffers from the disadvantage that the method of solution depends on solving a succession of Volterra integral equations; and, unless the boundary conditions specified along $D A B C$ are specially simple, we should not expect to go very far in the explicit carrying out of the solution by this method. It would, therefore, be useful if an alternative method of solution can be devised which will eliminate the need of solving integral equations and reduce the practical problem to one involving (at most!) only quadratures. It is remarkable that this can actually be accomplished by constructing suitable Green's functions and applying Green's theorem to contours, such as $R A S$ and $P Q B$.

39. The Green's functions $C\left(x, y ; x_{1}, y_{1}\right)$ and $\Gamma\left(x, y ; x_{1}, y_{1}\right)$. It is found that Green's functions which are appropriate for the solution of the boundary value problems of the type formulated in $\$ 38$ are $C\left(x, y ; x_{1}, y_{1}\right)$ and $\Gamma\left(x, y ; x_{1}, y_{1}\right)$, defined as follows:

$C\left(x, y ; x_{1}, y_{1}\right)$ is a solution of the hyperbolic equation (262) which satisfies the boundary conditions

$$
C\left(x_{1}, y ; x_{1}, y_{1}\right)=1
$$

and

$$
C\left(x, x_{1}+y_{1}-x ; x_{1}, y_{1}\right)=1 \quad\left(x \geqq x_{1}\right) .
$$

In other words, if $O$ represents the point $\left(x_{1}, y_{1}\right), O B$ the characteristic $x+y=x_{1}+y_{1}$ through $O$ and $O A$ the line through $O$ parallel to the $y$-axis, then the boundary conditions require that $C$ take the value 1 along both $O A$ and $O B$ (see Fig. 3). A solution satisfying these boundary conditions can be found explicitly. It is given by

$$
\begin{aligned}
C\left(x, y ; x_{1}, y_{1}\right)= & \cos \left(x-x_{1}\right)+\frac{1}{2}\left(x-x_{1}\right) \int_{0}^{\pi} J_{0}\left(\left[x-x_{1}\right] \sin \vartheta\right) \\
& \times I i_{1}\left(y_{1}-y-\left[x-x_{1}\right] \cos \vartheta\right) \sin \vartheta d \vartheta,
\end{aligned}
$$

where $J_{0}$ denotes the Bessel function of order zero and $I i_{1}(z)$ the "Bessel integral"

$$
I i_{1}(z)=\int_{0}^{z} \frac{I_{1}(t)}{t} d t
$$

$\left(I_{1}(t)\right.$ denotes the Bessel function of order 1 for a purely imaginary argument.)

The second function, $\Gamma\left(x, y ; x_{1}, y_{1}\right)$, is defined in terms of 
$C\left(x, y ; x_{1}, y_{1}\right)$ according to the formula

$$
\Gamma\left(x, y ; x_{1}, y_{1}\right)=\frac{\partial C}{\partial x}-\frac{\partial C}{\partial y} .
$$

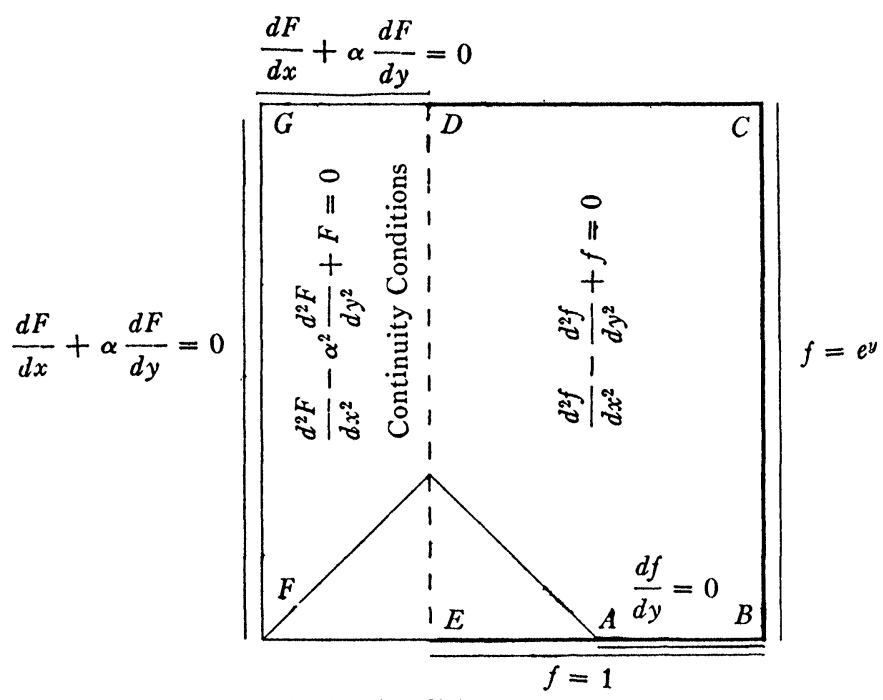

Continuity Conditions:

$$
\begin{gathered}
e^{-y} f=e^{y / \alpha} \cdot \alpha \\
e^{-\nu}\left(\frac{d f}{d x}-\frac{d f}{d y}\right)=e^{y / \alpha}\left(\frac{d F}{d r}+\alpha \frac{d F}{d y}\right)
\end{gathered}
$$

FIG. 4.

40. Some further boundary problems. The boundary value problem formulated in $\$ 38$ does not exhaust the type of problems which occur in theory of radiative transfer in moving atmospheres. However, no progress has so far been made in the solution of these other problems. It may, therefore, be of particular interest to describe here the nature of these more complex boundary value problems.

A typical problem is to solve (see Fig. 4)

$$
\frac{\partial^{2} F}{\partial x^{2}}-\alpha^{2} \frac{\partial^{2} F}{\partial y^{2}}+F=0 \quad(\alpha=\text { constant }),
$$

for $F$ in the rectangular strip $F E D G\left(0 \leqq x \leqq x_{2}, 0 \leqq y \leqq y_{1}\right)$, and 


$$
\frac{\partial^{2} f}{\partial x^{2}}-\frac{\partial^{2} f}{\partial y^{2}}+f=0,
$$

for $f$ in the rectangular strip $E B C D\left(x_{2} \leqq x \leqq x_{1}, 0 \leqq y \leqq y_{1}\right)$ satisfying the following boundary conditions:

Along $F G\left(x=0,0 \leqq y \leqq y_{1}\right)$ and $G D\left(y=y_{1}, 0 \leqq x \leqq x_{2}\right)$ relations of the form

$$
\left(\frac{\partial F}{\partial x}+\alpha \frac{\partial F}{\partial y}\right)_{x=0}=\phi(y) \quad\left(0 \leqq y \leqq y_{1}\right)
$$

and

$$
\left(\frac{\partial F}{\partial x}+\alpha \frac{\partial F}{\partial y}\right)_{y=y_{1}}=\psi(x) \quad\left(0 \leqq x \leqq x_{2}\right)
$$

are specified where $\phi(y)$ and $\psi(x)$ are two known functions. Along $E B\left(y=0, x_{2} \leqq x \leqq x_{1}\right)$ and $B C\left(x=x_{1}, 0 \leqq y \leqq y_{1}\right) f$ is given, while along the part $A B\left(x^{*}=x_{2}(1+\alpha) \leqq x \leqq x_{1}, y=0\right)$ of the $x$-axis, the derivative $\partial f / \partial y$ is also given. And, finally, along $E D\left(x=x_{2}, 0 \leqq y \leqq y_{1}\right)$ certain "continuity conditions" of the type

$$
f\left(x_{2}, y\right)=Q(y) F\left(x_{2}, y\right) \quad\left(0 \leqq y \leqq y_{1}\right)
$$

and

$$
\left(\frac{\partial f}{\partial x}-\frac{\partial f}{\partial y}\right)_{x=x_{2}}=Q(y)\left(\frac{\partial F}{\partial x}+\alpha \frac{\partial F}{\partial y}\right)_{x=x_{2}} \quad\left(0 \leqq y \leqq y_{1}\right)
$$

are specified, where $Q(y)$ is a known function.

It would be of considerable interest to know how such boundary value problems can be solved. (In Fig. 4 the particular boundary conditions which occur in a specific problem are indicated.)

41. Concluding remarks. In concluding, I may recall what I said at the beginning, namely, that the advance of a branch of theoretical physics often leads to the creation of a new mathematical discipline. I think it may be conversely said, with almost equal truth, that the creation of a new mathematical discipline is often the sign that the particular branch of theoretical physics has reached maturity. I hope I have given you the impression that theoretical astrophysics has now come of age.

Since the lecture was given in December, the mathematical theory of radiative transfer has advanced along several directions and in this Addendum we shall briefly summarize the results of these newer investigations. The particular sections of the lecture to which these 
advances refer are indicated by the numbering of the paragraphs which follow; however, $\$ 42$ breaks new ground not covered by the lecture.

11a. Elliptically polarized radiation field. In $\$ 11$ we outlined how the equations of transfer for a partially plane-polarized radiation field can be formulated and gave the explicit form of these equations for the particular case of Rayleigh scattering. It is not difficult to extend this discussion to include the case of a general elliptic polarization of the radiation field. In this latter case, we must consider, in addition to the intensities $I_{l}$ and $I_{r}$ in two directions at right angles to each other in the plane of the electric and the magnetic vectors, the two further quantities

$$
U=\left(I_{l}-I_{r}\right) \tan 2 \chi \text { and } V=\left(I_{l}-I_{r}\right) \tan 2 \beta \sec 2 \chi
$$

where $\chi$ denotes the inclination of the plane of polarization to the direction to which $l$ refers and $-\pi / 2 \leqq \beta \leqq+\pi / 2$ is an angle the tangent of which is equal to the ratio of axes of the ellipse characterizing the state of polarization. (The sign of $\beta$ depends on whether the polarization is right-handed $(+)$ or left-handed $(-)$.) And the rule of composition (due to Stokes) is that a mixture of several independent streams of polarized light is characterized by values of the parameters $I_{l}, I_{r}, U$ and $V$ which are the sums of the respective parameters of the individual streams. With this rule of composition, the equations of transfer for $I_{l}, I_{r}, U$ and $V$ can be formulated in terms of the basic laws of single scattering.

For the case of Rayleigh scattering, it is found that the equations of transfer for $I_{l}, I_{r}$ and $U$ are of exactly the same forms as when, only, plane polarization is contemplated; that is, in the case of Rayleigh scattering, the equations are reducible. Moreover, it is found that for Rayleigh scattering, $V$ is simply scattered in accordance with a phase function $3 / 2 \cos \Theta$; and the exact solution of the equation for $V$ therefore presents no difficulty.

23a. The functional equation relating the law of darkening and the scattering function for semi-infinite plane-parallel atmospheres. In $\$ 23$ we have remarked on the remarkable relationships between the angular distributions of the emergent radiation in the problem with a constant net flux and the law of diffuse reflection. It has since been possible to trace the origin of these relationships: they arise simply in consequence of the invariance of the emergent radiation $I(0, \mu)$ (in the problem with a constant net flux) to the addition (or removal) of layers of arbitrary thickness to (or from) the atmosphere. The mathe- 
matical expression of this invariance is that the outward radiation $I(\tau,+\mu)(0<\mu<1)$ at any level $\tau$ differs from the emergent radiation $I(0, \mu)$ only on account of the fact that at $\tau$ there is an inward directed radiation field specified by $I\left(\tau,-\mu^{\prime}\right)\left(0<\mu^{\prime}<1\right)$ which will be reflected by the atmosphere below $\tau$ by the law of diffuse reflection of a semi-infinite atmosphere. In other words, we must have

$$
I(\tau,+\mu)=I(0, \mu)+\frac{1}{2 \mu} \int_{0}^{1} S^{(0)}\left(\mu, \mu^{\prime}\right) I\left(\tau,-\mu^{\prime}\right) d \mu^{\prime},
$$

where

$$
S^{(0)}\left(\mu, \mu^{\prime}\right)=\frac{1}{2 \pi} \int_{0}^{1} S\left(\mu, \phi ; \mu^{\prime}, \phi^{\prime}\right) d \phi^{\prime}
$$

is the azimuth independent term in the scattering function $S(\mu, \phi$; $\mu^{\prime}, \phi^{\prime}$ ) (cf. equation (26)).

Differentiating equation (278) with respect to $\tau$ and passing to the limit $\tau=0$, we obtain

$$
\left[\frac{d I(\tau,+\mu)}{d \tau}\right]_{\tau=0}=\frac{1}{2 \mu} \int_{0}^{1} S^{(0)}\left(\mu, \mu^{\prime}\right)\left[\frac{d I\left(\tau,-\mu^{\prime}\right)}{d \tau}\right]_{\tau=0} d \mu^{\prime} .
$$

On the other hand, from the equation of transfer

$$
\mu \frac{d I}{d \tau}=I(\tau, \mu)-B(\tau, \mu)
$$

where $B(\tau, \mu)$ is the source function appropriate for the problem (see equations I, II and VI) we conclude that

$$
\left[\frac{d I(\tau,+\mu)}{d \tau}\right]_{\tau=0}=\frac{1}{\mu}[I(0, \mu)-B(0, \mu)],
$$

and

$$
\left[\frac{d I\left(\tau,-\mu^{\prime}\right)}{d \tau}\right]_{\tau=0}=\frac{1}{\mu^{\prime}} B\left(0,-\mu^{\prime}\right) .
$$

Now combining equations (280), (282) and (283), we obtain

$$
I(0, \mu)=B(0, \mu)+\frac{1}{2} \int_{0}^{1} S^{(0)}\left(\mu, \mu^{\prime}\right) B\left(0,-\mu^{\prime}\right) \frac{d \mu^{\prime}}{\mu^{\prime}},
$$

which is a functional equation relating $I(0, \mu)$ and $S^{(0)}\left(\mu, \mu^{\prime}\right)$; it can be shown that it is precisely in consequence of this equation that the relationship between $I(0, \mu)$ and $S^{(0)}\left(\mu, \mu^{\prime}\right)$ noticed in $\$ 23$ arises. 
35a. Representation of $H(\mu)$ as a complex integral. According to equations (102) and (110)

$$
1-\sum_{j=1}^{n} \frac{a_{j}}{1-\mu_{j}^{2} / z^{2}} \equiv \frac{1}{H(z) H(-z)}
$$

where $H(z)$ is defined as usual in terms of the roots of the characteristic equation (see equation (49))

$$
1=\sum_{j=1}^{n} \frac{a_{j}}{1-\mu_{j}^{2} k^{2}} .
$$

The arguments leading to equation (285) ( $\$ 20)$ are seen to be sufficiently general to establish the identity

$$
\begin{aligned}
1-2 \sum_{j=1}^{n} \frac{a_{j} \Psi\left(\mu_{j}\right)}{1-\mu_{j}^{2} / z^{2}} & \equiv \frac{1}{H(z) H(-z)} \\
& =\frac{\prod_{\alpha}\left(1-k_{\alpha}^{2} z^{2}\right)}{\prod_{j}\left(1-z^{2} / \mu_{j}^{2}\right)},
\end{aligned}
$$

where $H(z)$ is now defined as in equation (126) in terms of the roots of the characteristic equation

$$
1=2 \sum_{j=1}^{n} \frac{a_{j} \Psi\left(\mu_{j}\right)}{1-k^{2} \mu_{i}^{2}}
$$

and $\Psi(\mu)$ has the same meaning as in equation (127).

Now let

$$
G(x)=\frac{1}{2 \pi i} \int_{-i \infty}^{i \infty} \log T(z) \frac{x d z}{z^{2}-x^{2}}
$$

where

$$
T(z)=1-2 z^{2} \sum_{j=1}^{n} \frac{a_{j} \Psi\left(\mu_{j}\right)}{z^{2}-\mu_{j}^{2}}=\frac{\bigwedge_{\alpha}\left(1-k_{\alpha}^{2} z^{2}\right)}{\prod_{j}\left(1-z^{2} / \mu_{j}^{2}\right)} .
$$

It is seen that defined in this manner $G(x)$ is regular for $R(x)>0$.

By evaluating the residue at the pole on the right

$$
\frac{1}{2 \pi i} \int_{-i \infty}^{i \infty} \log \left(1+\frac{z}{a}\right) \frac{x d z}{z^{2}-x^{2}}=-\frac{1}{2} \log \left(1+\frac{x}{a}\right)
$$


if $R(x)>0$ and $R(a)>0$. Similarly, by evaluating the residue at the pole on the left, we have

$$
\frac{1}{2 \pi i} \int_{-i \infty}^{i \infty} \log \left(1-\frac{z}{a}\right) \frac{x d z}{z^{2}-x^{2}}=-\frac{1}{2} \log \left(1+\frac{x}{a}\right) .
$$

Hence,

$$
\frac{1}{2 \pi i} \int_{-i \infty}^{i \infty} \log \left(1-\frac{z^{2}}{a^{2}}\right) \frac{x d z}{z^{2}-x^{2}}=-\log \left(1+\frac{x}{a}\right) .
$$

Accordingly

$$
\begin{aligned}
G(x) & =\frac{1}{2 \pi i} \int_{-i \infty}^{i \infty}\left\{\sum_{\alpha=1}^{n} \log \left(1-k_{\alpha}^{2} z^{2}\right)-\sum_{j=1}^{n} \log \left(1-\frac{z^{2}}{\mu_{j}^{2}}\right)\right\} \frac{x d z}{z^{2}-x^{2}} \\
& =-\sum_{\alpha=1}^{n} \log \left(1+x k_{\alpha}\right)+\sum_{j=1}^{n} \log \left(1+\frac{z}{\mu_{j}}\right) \\
& =\log H(z) .
\end{aligned}
$$

We have thus shown that

$$
\log H(x)=\frac{1}{2 \pi i} \int_{-i \infty}^{i \infty} \log T(z) \frac{x d z}{z^{2}-x^{2}} .
$$

From the representation (295) of the $H$-function as a complex integral, it would appear that the solution of the functional equation

$$
H(\mu)=1+\mu H(\mu) \int_{0}^{1} \frac{H\left(\mu^{\prime}\right)}{\mu+\mu^{\prime}} \Psi\left(\mu^{\prime}\right) d \mu^{\prime}
$$

has the representation (cf. Theorem $2, \S 25$ )

$$
\log H(\mu)=\frac{1}{2 \pi i} \int_{-i \infty}^{i \infty} \log T(z) \frac{\mu d z}{z^{2}-\mu^{2}}
$$

where, now (see equation (290)),

$$
T(z)=1-2 z^{2} \int_{0}^{1} \frac{\Psi(\mu) d \mu}{z^{2}-\mu^{2}} .
$$

Our arguments do not of course establish rigorously the representation (297) of the solution of equation (296). However, Professor E. C. Titchmarsh, with whom I have corresponded, has kindly communicated to me a rigorous demonstration of the representation (297) by one of his colleagues, Mr. M. M. Crum. 
42. The theory of radiative transfer in atmospheres of finite optical thicknesses. In the lecture attention was directed almost exclusively to transfer problems in semi-infinite plane-parallel atmospheres. The extension of this theory to the study of the transfer of radiation in plane-parallel atmospheres of finite optical thicknesses raises problems of a higher order of difficulties; these difficulties arise principally from the circumstance that boundary conditions have to be explicitly satisfied on both sides of the atmosphere. Thus, if we consider an atmosphere of optical thickness $\tau_{1}(<\infty)$, we are interested, for example, in solutions of the equations of transfer I-VII which satisfy the boundary conditions

$$
I(0,-\mu)=0 \quad(0<\mu<1),
$$

and

$$
I\left(\tau_{1},+\mu\right)=0 \quad(0<\mu<1) .
$$

However, in analogy with the theory of semi-infinite atmospheres, we may expect that in the case of finite atmospheres, also, the angular distributions of the emergent radiations can be expressed in terms of functions (like $H(\mu)$ ) which will be explicitly known in any finite approximation and which, in the limit of infinite approximation, will become solutions of functional equations of a standard form. It now appears that this reduction can in fact be achieved and that the pair of functional equations

$$
X(\mu)=1+\mu \int_{0}^{1} \frac{X(\mu) X\left(\mu^{\prime}\right)-Y(\mu) Y\left(\mu^{\prime}\right)}{\mu+\mu^{\prime}} \Psi\left(\mu^{\prime}\right) d \mu^{\prime},
$$

and

$$
Y(\mu)=e^{-\tau_{1} / \mu}+\mu \int_{0}^{1} \frac{Y(\mu) X\left(\mu^{\prime}\right)-X(\mu) Y\left(\mu^{\prime}\right)}{\mu-\mu^{\prime}} \Psi\left(\mu^{\prime}\right) d \mu^{\prime},
$$

where $\Psi(\mu)$ is an even polynomial in $\mu$ satisfying the condition

$$
\int_{0}^{1} \Psi(\mu) d \mu<\frac{1}{2}
$$

plays the same basic role in the theory of atmospheres of finite optical thicknesses as the functional equation

$$
H(\mu)=1+\mu H(\mu) \int_{0}^{1} \frac{H\left(\mu^{\prime}\right)}{\mu+\mu^{\prime}} \Psi\left(\mu^{\prime}\right) d \mu^{\prime}
$$

played in the theory of semi-infinite atmospheres. And just as 


$$
H(\mu)=\frac{1}{\mu_{1} \cdots \mu_{n}} \frac{\prod_{i}\left(\mu+\mu_{i}\right)}{\prod_{\alpha}\left(1+k_{\alpha} \mu\right)},
$$

where the $k_{\alpha}$ 's are the positive roots of the characteristic equation

$$
1=2 \sum_{j=1}^{n} \frac{a_{j} \Psi\left(\mu_{j}\right)}{1-k^{2} \mu_{j}^{2}},
$$

provides a rational approximation to the solution of equation (304), so also the functions $X(\mu)$ and $Y(\mu)$ defined in the manner of the following equations provides an approximation to the solution of equations (301) and (302):

$$
\begin{array}{r}
X(\mu)=\frac{(-1)^{n}}{\mu_{1} \cdots \mu_{n}} \frac{1}{\left[C_{0}^{2}(0)-C_{1}^{2}(0)\right]^{1 / 2}} \frac{1}{W(\mu)} \\
\cdot\left[P(-\mu) C_{0}(-\mu)-e^{-\tau_{1} / \mu} P(\mu) C_{1}(\mu)\right] \\
Y(\mu)=\frac{(-1)^{n}}{\mu_{1} \cdots \mu_{n}} \frac{1}{\left[C_{0}^{2}(0)-C_{1}^{2}(0)\right]^{1 / 2}} \frac{1}{W(\mu)} \\
\cdot\left[e^{-\tau_{1} / \mu} P(\mu) C_{0}(\mu)-P(-\mu) C_{1}(-\mu)\right]
\end{array}
$$

where

$$
\begin{aligned}
& P(\mu)=\prod_{i=1}^{n}\left(\mu-\mu_{i}\right), \quad W(\mu)=\prod_{\alpha=1}^{n}\left(1-k_{\alpha}^{2} \mu^{2}\right), \\
& C_{0}(\mu)=\sum_{2^{n-1} \text { terms }} \epsilon_{2 l}^{(0)} \frac{\prod_{i=1}^{2 l} \prod_{m=1}^{n-2 l}\left(k_{r_{i}}+k_{s_{m}}\right)}{\prod_{i=1}^{2 l} \prod_{m=1}^{n-2 l}\left(k_{r_{i}}-k_{s_{m}}\right)} \\
& \times \prod_{i=1}^{2 l} \xi_{r_{i}}\left(1+k_{r_{i}} \mu\right) \prod_{m=1}^{n-2 l} \eta_{s_{m}}\left(1-k_{s_{m}} \mu\right), \\
& C_{1}(\mu)=\sum_{2^{n-1} \text { terms }} \epsilon_{\epsilon_{2 l+1}} \prod_{\prod_{i=1}^{2 l+1}}^{\prod_{i=1}^{2 l+1} \prod_{m=1}^{n-2 l-1}\left(k_{r_{i}}+k_{s_{m}}\right)} \\
& \times \prod_{i=1}^{2 l+1} \xi_{r_{i}}\left(1+k_{r_{i}} \mu\right) \prod_{m=1}^{n-2 l-1} \eta_{r_{i}}\left(1-k_{s_{m}} \mu\right)
\end{aligned}
$$


In equations (310) and (311), $\left(r_{1}, \cdots, r_{j}\right)$ and $\left(s_{1}, \cdots, s_{n-j}\right)$ are $j$, respectively, $n-j$ distinct integers from the set $(1,2, \cdots, n)$,

$$
\begin{gathered}
\epsilon_{2 l}^{(0)}=\left\{\begin{array}{l}
+1 \text { for even integers of the form } 4 l, \\
-1 \text { for even integers of the form } 4 l+2,
\end{array}\right. \\
\epsilon_{2 l+1}^{(1)}=\left\{\begin{array}{l}
+1 \text { for odd integers of the form } 4 l+1, \\
-1 \text { for odd integers of the form } 4 l+3,
\end{array}\right.
\end{gathered}
$$

and

$$
\begin{array}{r}
\xi_{\alpha}=e^{k_{\alpha} \tau_{1} / 2} P\left(-1 / k_{\alpha}\right) \text { and } \eta_{\alpha}=e^{-k_{\alpha} \tau_{1} / 2} P\left(+1 / k_{\alpha}\right) \\
(\alpha=1, \cdots, n) .
\end{array}
$$

Finally, it should be noted that the definitions of $C_{0}(\mu)$ and $C_{1}(\mu)$ according to equations (307) and (308) are valid only in even orders of approximation; in odd orders the role of $C_{0}$ and $C_{1}$ should be interchanged.

Moreover, there exist also functional equations for the scattering and the transmission functions for the problem of diffuse reflection and transmission by atmospheres of finite optical thicknesses. These equations arise from general invariances of the type considered in $\S \S 28,29$ and $23 \mathrm{a}$ and lead to a whole new class of systems of functional equations which can all be reduced to the solution of pairs of functional equations of the form (301) and (302). It is therefore apparent that the study of the transfer of radiation in atmospheres of finite optical thicknesses will lead to the development of a mathematical theory at least as extensive as the one described in the lecture in the context of semi-infinite atmospheres.

\section{REFERENCES}

The following papers and monographs substantially form the basis of the report given in the lecture. The list is not intended to be a complete bibliography of the subject of the transfer of radiation in stellar atmospheres.

\section{A. Ambarzumian}

C. R. (Doklady) Acad. Sci. URSS. vol. 38 (1943) p. 257.

Russian Astronomical Journal vol. 19 (1942).

Journal of Physics of the Academy of Sciences of the U.S.S.R. vol. 8 (1944) p. 65.

S. Chandrase khar

Astrophysical Journal vol. 99 (1944) p. 180; vol. 100 (1944) pp. 76, 117, 355; vol. 101 (1945) pp. 95, 328, 348; vol. 102 (1945) p. 402; vol. 103 (1946) pp. 165, 351; vol. 104 (1946) pp. 110, 191; vol. 105 (1947) pp. 151, 164, 424, 435, 441, 461. (Further papers in this series are in course of publication in the same journal.) 
Reviews of Modern Physics vol. 17 (1945) p. 138.

Proc. Chambridge Philos. Soc. vol. 42 (1946) p. 250.

E. HopF

Mathematical problems of radiative equilibrium, Cambridge Mathematical Tracts, No. 31, Cambridge, England, 1934.

G. MÜNCH

Astrophysical Journal vol. 104 (1946) p. 87.

LORD RAYLEIGH

Scientific papers of Lord Rayleigh, vol. 1, Cambridge, England, 1899, pp. 87, 104, 518.

Sir GeORge Stokes

Trans. Cambridge Philos. Soc. vol. 9 (1852) p. 399.

M. TUberg

Astrophysical Journal vol. 103 (1946) p. 145.

G. C. WICK

Zeitschrift für Physik vol. 120 (1943) p. 702.

Yerkes Observatory, University of Chicago 